\title{
Black hole spin constraints on the mass spectrum and number of axionlike fields
}

\author{
Matthew J. Stott ${ }^{1, *}$ and David J. E. Marsh ${ }^{2, \dagger}$ \\ ${ }^{1}$ Theoretical Particle Physics and Cosmology Group, Department of Physics, King's College London, \\ University of London, Strand, London, WC2R 2LS, United Kingdom \\ ${ }^{2}$ Institüt für Astrophysik, Georg-August Universität, \\ Friedrich-Hund-Platz, 1, D-37077 Göttingen, Germany
}

(Received 8 June 2018; published 9 October 2018)

\begin{abstract}
Astrophysical observations of spinning black holes, which span $5 M_{\odot} \lesssim M_{\mathrm{BH}} \lesssim 5 \times 10^{8} M_{\odot}$, can be used to exclude the existence of certain massive bosons via the superradiance phenomenon. In this work, we explore for the first time how these measurements can be used to constrain properties of statistical distributions for the masses of multiple bosonic fields. Quite generally, our methodology excludes $N_{\text {ax }} \gtrsim 30$ scalar fields with a range of mass distribution widths and central values spanning many orders of magnitude. We demonstrate this for the specific example of axions in string theory and M-theory, where the mass distributions in certain cases take universal forms. We place upper bounds on $N_{\text {ax }}$ for certain scenarios of interest realized approximately as mass distributions in M-theory, including the QCD axion, grand unified theories, and fuzzy dark matter.
\end{abstract}

DOI: $10.1103 /$ PhysRevD.98.083006

\section{INTRODUCTION}

The Penrose process [1] allows bosonic waves infalling into a Kerr black hole $(\mathrm{BH})$ to emerge with more energy than incident upon entry at the horizon, in exact analogy to other superradiant processes in physics, such as Cherenkov radiation. If the bosons can be confined around the $\mathrm{BH}$ by a mirror, then this amplification process continues without limit leading to Press and Teukolsky's "black hole bomb" scenario $[2,3]$ which was initially quantitatively studied in Ref. [4]. Massive bosonic fields on a Kerr spacetime possess hydrogenic bound states. In this case the potential barrier provided by the particle mass can play the role of the mirror, leading to a natural realization of the $\mathrm{BH}$ superradiance process for massive bosons in orbits around astrophysical BHs (see Ref. [5] for a review). The historic Laser Interferometry Gravitational-Wave Observatory (LIGO) observations of gravitational waves from the binary coalescence of astrophysical BHs has ushered in a new era of interest in $\mathrm{BH}$ physics [6]. Gravitational wave data can be used to infer the mass and spin of the two BHs in the binary. LIGO could potentially detect the existence of

\footnotetext{
matthew.stott@kcl.ac.uk

†david.marsh@uni-goettingen.de
}

Published by the American Physical Society under the terms of the Creative Commons Attribution 4.0 International license. Further distribution of this work must maintain attribution to the author(s) and the published article's title, journal citation, and DOI. Funded by SCOAP ${ }^{3}$. many hundreds of such events, accurately determining the mass and spin distribution of BHs. The future of $\mathrm{BH}$ superradiance constraints derived from LIGO, the growing global network of GW observatories, and future space-based missions, is extremely promising as a probe of fundamental physics [7-11].

The ability to constrain ultralight bosonic fields from $\mathrm{BH}$-scalar condensate systems come in the form of two phenomena. It may be possible to identify the presence of scalar clouds in the vicinity of BHs as emission sources of monochromatic gravitational waves (GWs). The signal frequency, $f \sim \mu_{\mathrm{ax}} / \pi$, with boson mass, $\mu_{\mathrm{ax}}$ could potentially be detected by either ground- or space-based GW observatories and proposes to be an exciting methodology to enhance constraints on the mass bounds for bosonic fields. This subject has been extensively discussed in Refs. [12-15]. The second phenomenon of interest, and the subject of this work, is the spin-down of astrophysical BHs. If the superradiance rate is faster than any other astrophysical process affecting the $\mathrm{BH}$ mass, $M_{\mathrm{BH}}$, and dimensionless spin, $a_{*}$, then the $\mathrm{BH}$ superradiance process can efficiently reduce these quantities. This occurs when the boson Compton wavelength is of the order of the gravitational radius of the $\mathrm{BH}$. Thus, if a massive boson exists, then astrophysical $\mathrm{BHs}$ of particular values in the $\left(M_{\mathrm{BH}}, a_{*}\right)$ "Regge plane" (which, according to the no-hair theorems, gives a complete description of spinning BHs) should be absent in observations. The masses and spins of a large number of astrophysical BHs have been measured, often incorporating either $\mathrm{x}$-ray reflection 
spectroscopy or continuum-fitting methods (see Table I for $\mathrm{BH}$ parameter measurements and corresponding references). These measurements can be used to probe the possible existence of massive bosons [16,17]. BH superradiance constraints apply to a range of particle physics models, including a possible mass for the graviton or the photon [18] (and indeed to the photon plasma mass near the $\mathrm{BH}$ ), as well as to exotic particles, such as massive vector (Proca) fields [8], massive spin-two fields [19], and axionlike particles and other massive scalars $[12,20,21]$.

BH superradiance excludes two separate ranges of axion masses, $\mu_{\mathrm{ax}}$. Stellar mass BHs exclude $7 \times 10^{-14} \mathrm{eV}<$ $\mu_{\mathrm{ax}} / \mathrm{eV}<2 \times 10^{-11}$ at the $95 \%$ C.L., while supermassive BHs (SMBHs) exclude $7 \times 10^{-20} \mathrm{eV}<\mu_{\mathrm{ax}} / \mathrm{eV}<1 \times 10^{-16}$ at the $95 \%$ C.L. These limits apply strictly in the regime of zero self-coupling. Assuming a self-coupling derived from a standard instanton potential, they apply for axions with decay constants $f_{a} \gtrsim 10^{14} \mathrm{GeV}$ [21], a limit we assume throughout the remainder of this work.

These are powerful and generic exclusions, but they leave many axion models of interest unconstrained. Stellar BHs are too heavy to place constraints on the QCD axion [56-58] possessing a decay constant far below the Planck scale [21]. "Fuzzy dark matter (DM)" with $\mu_{\mathrm{ax}} \approx 10^{-22} \mathrm{eV}$ [59-63], which has novel effects on the formation of galaxies, is too light to make predictions about the spin distribution of SMBHs with $M_{\mathrm{BH}}<10^{9} M_{\odot}$ that inhabit the centers of galaxies. Finally, the axion mass scale associated with grand unification theories (GUTs) in M-theory, $\mu_{\mathrm{ax}} \approx 10^{-15} \mathrm{eV}$ [64] is in the "desert" of intermediate mass BHs (IMBHs) which so far have not been observed. There is hope, however, since each of these models is only a small logarithmic distance from the $\mathrm{BH}$ superradiance constrained regions, while axion models typically have a spectrum spanning many orders of magnitude $[20,65]$. All previous studies of $\mathrm{BH}$ superradiance constraints on bosons have focused on the range of excluded masses assuming the existence of a single new bosonic field. In the present work we assess, for the first time, what constraints can be drawn on the properties of axion mass distributions from $\mathrm{BH}$ superradiance.

String theory and M-theory predict that there should be a large number of as-yet-undiscovered light bosonic degrees of freedom, including hidden $U(1)$ gauge fields, moduli, and axions [20,64,66-69]. The number of axion fields depends on the details of the compactification of the six-/ seven-extra-dimensional space determining the required $3+1$ spacetime dimensions. Typical numbers of axions are of order 30 in Calabi-Yau compactifications [70,71], with a similar expectation for $G_{2}$ manifolds [72-78], although certain flux compactifications could contain upwards of $10^{5}$ axions [79]. Significant progress can also be made towards general predictions since the mass distributions of large numbers of axions possess universal properties thanks to results from random matrix theory (RMT) [65,80-85].

Any string or M-theory model that realizes one of the models of interest (QCD axion, fuzzy DM, or GUTs) will likely contain a distribution of masses around this value. Even a small spread on a logarithmic scale could lead to strong constraints on the model. The central observation of the present work is that, simply from the statistical overlap between a mass distribution and the $\mathrm{BH}$ superradiance bounds, it is possible to place constraints on the allowed mass distributions of axions. Furthermore, these constraints get increasingly more stringent as the number of axions increases, placing upper bounds on the number of axionlike fields. Consider the following toy model. In Ref. [20] it was suggested that axion masses have a log-flat distribution from the Planck scale to the Hubble scale, covering approximately 60 orders of magnitude. The $\mathrm{BH}$ superradiance constraints cover approximately 4 orders of magnitude. Assuming independent and identically distributed draws from the log-flat distribution, this naive model of the axiverse is excluded with probability $P=1-(56 / 60)^{N}$, which is greater than $95 \%$ C.L. if $N_{\mathrm{ax}} \geq 44$. Clearly, the model with a log-flat prior on the axion mass is excluded by BH superradiance for large numbers of fields. The exclusion is a function of the upper and lower bounds on the mass spectrum. The constraint gets considerably stronger if the upper bound is below the Planck scale, and vanishes if the distribution does not extend below about $10^{-11} \mathrm{eV}$. Such a truncated spectrum, on the other hand, cannot realize many of the models of interest discussed above.

Fortunately for phenomenologists, the mass distributions arising from RMT models are not log flat from the Hubble scale to the Planck scale. The log-normal distribution, centered on a particular mean mass, $\mu_{\mathrm{ax}}$, and with a variance $\sigma^{2}$, provides a useful benchmark, covering different types of models. For small $\sigma$, it resembles a degenerate spectrum, large $\sigma$ is approximately log flat, and intermediate values of $\sigma$ are statistically similar to eigenvalue distributions found in RMT and M-theory. Figure 1 summarizes our conclusions, showing the allowed number of axionic fields drawn from log-normal distributions as a function of the width and central value.

The structure of this work is as follows. Section II contains a brief review of $\mathrm{BH}$ superradiance along with the Regge plane and BH spin measurements, while Sec. III overviews our models for the axion mass matrix and collects our BH data. In Sec. IV we present constraints on axion mass spectra from BH mass and spin measurements under a frequentist framework. We first reproduce the known single-field results and then move on to considering mass distributions. We conclude our work in Sec. V. Appendix A describes our statistical methods, which we believe are somewhat novel in this context. Appendix B collects results from Ref. [65] on the axion mass matrix and RMT. 


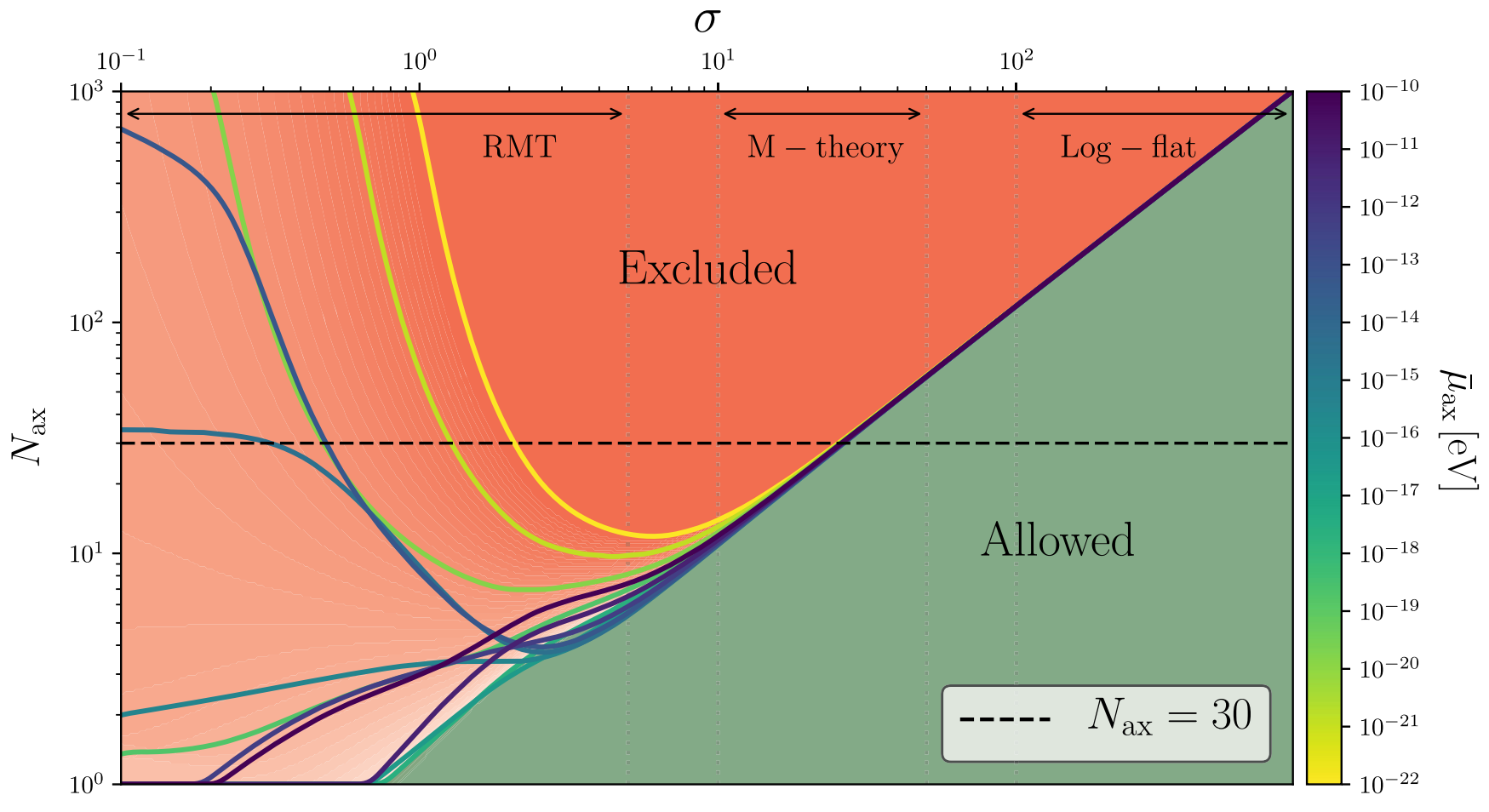

FIG. 1. Summary of results displaying contours for the $95 \%$ exclusion regions for log-normal axion mass distributions as a function of the width, $\sigma$, and number of fields, $N_{\mathrm{ax}}$, for various central masses, $\bar{\mu}_{\mathrm{ax}}$. Regions above the contours are excluded. Certain ranges of $\sigma$ correspond closely to RMT and M-theory mass spectra, and can also be used to approximate the log-flat spectrum. For $1 \lesssim \sigma \lesssim 20$, $N_{\text {ax }} \geq 30$ is excluded for an extremely wide range of central masses. Constraints neglect axion self-interactions and apply approximately in the limit of large decay constants, $f_{a} \gtrsim 10^{14} \mathrm{GeV}$.

\section{BLACK HOLE SUPERRADIANCE}

\section{A. Scalar fields on Kerr background}

The action for $N$ real scalar fields $\Psi_{i}$ with masses $\mu_{i}$ takes the form

$$
S=\int d^{4} x \sqrt{-g} \sum_{i}\left(-\frac{1}{2} \nabla_{\mu} \Psi_{i} \nabla^{\mu} \Psi_{i}-\frac{1}{2} \mu_{i} \Psi_{i}^{2}\right),
$$

where $\nabla_{\mu}$ is the covariant derivative on the spacetime with metric $g$. The metric is assumed to be the Kerr metric for a spinning $\mathrm{BH}$ where the 3+1-dimensional spacetime region outside the horizon of a rotating Kerr $\mathrm{BH}$ is described by the invariant line element, $d s^{2}=g_{\alpha \beta} d x^{\alpha} d x^{\beta}$ which, using the standard Boyer-Lindquist coordinates $(t, r, \theta, \phi)$ and metric signature $[-,+,+,+]$, takes the form

$$
\begin{aligned}
d s_{\mathrm{Kerr}}^{2}= & -\left(1-\frac{2 M_{\mathrm{BH}} r}{\Sigma}-\right) d t^{2}-\frac{4 M_{\mathrm{BH}} a r \sin ^{2} \theta}{\Sigma} d t d \phi \\
& +\frac{\Sigma}{\Delta} d r^{2}+\Sigma d \theta^{2}+\frac{\left(r^{2}+a^{2}\right)^{2}-a^{2} \Delta \sin ^{2} \theta}{\Sigma} \sin ^{2} \theta d \phi^{2},
\end{aligned}
$$

which is invariant under time translations, as it possesses a Killing vector. This geometry is taken as a background and the characteristic behavior of each $\mathrm{BH}$ horizon for a fixed value of the dimensionless spin is displayed in Fig. 2.

The superradiant process leads to time dependence of the $\mathrm{BH}$ mass and spin, but the structure of the metric does not change due to backreaction. It is known for single-field superradiance that the backreaction of the scalar condensate on the Kerr geometry is small. This is because, although the cloud can obtain a large mass, it is distributed over a large volume compared to the $\mathrm{BH}$, leading to low scalar energy density (and thus a low source of curvature) in the cloud [86]. Concerns that backreaction is a more severe problem with large numbers of fields as opposed to dealing with a single field can be alleviated considering the properties of the scalar cloud. The gravitational backreaction is a function of $M_{S} / M_{\mathrm{BH}}$, where $M_{S}$ is the total mass in the scalar cloud. There is a maximum value of $M_{S}$ independent of the number of axion fields, which is determined by the $\mathrm{BH}$ mass at the initial spin, $M_{\mathrm{BH}}\left(a_{*}\right)$, and the irreducible mass after all the spin has been extracted, $M_{\mathrm{BH}}\left(a_{*}=0\right)$. $N_{\text {ax }}$ fields cannot extract any more total mass than a single field, and for resonant modes the cloud size is of the same order of magnitude for all the fields, and therefore gravitational backreaction is not enhanced to a greater severity than the single-field case. Nonlinearities coming from axion interactions, on the other hand, can increase with the number of fields. We discuss this briefly later. 


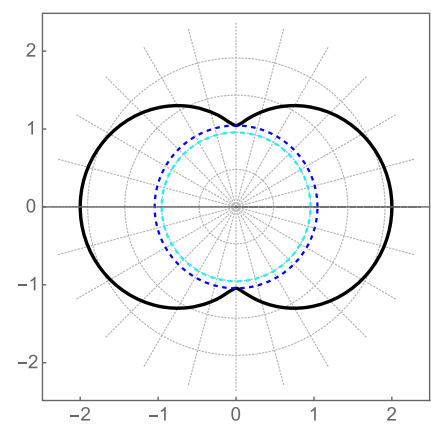

(a) $\mathrm{xz} / a_{*}=0.999$.

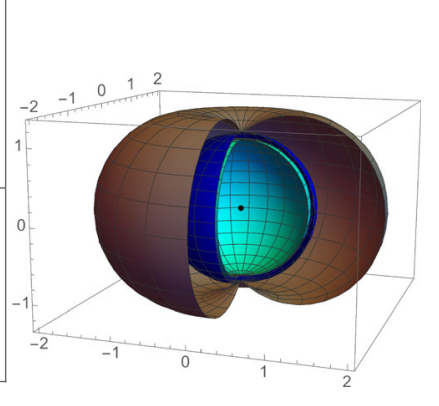

(b) xyz / $a_{*}=0.999$.
FIG. 2. Kerr BH horizons in the $x-z$ plane and $x-y-z$ volume for a fixed value of the dimensionless spin parameter, $a_{*}$ approaching the limit for an extremal $\mathrm{BH}$. The $x$ axis represents the radial distance from the $\mathrm{BH}$ in polar coordinates. The solid black line/ surface defines the ergoregion, while the dashed/solid blue and cyan line/surface represent the outer and inner horizons respectively in the $x-z$ plane $/ x-y-z$ volume. The two hypersurfaces of the event horizon and the ergosphere meet at the colatitude pole of 0 degrees.

Thus, neglecting the self-interactions, each field $\Psi_{i}$ evolves independently on the fixed background. In this separable limit, the total rate of the superradiant process is given simply by the sum of the single-field rates,

$$
\Gamma_{\mathrm{tot}}=\sum_{i} \Gamma_{i}
$$

which defines the superradiance phenomenon for multiple fields.

\section{B. Superradiance and spin-down rates}

Astrophysical BHs with a mass $M_{\mathrm{BH}}$ and spin $J=a M_{\mathrm{BH}}$ will spin down via superradiant instabilities extracting energy and angular momentum [5,12], forming very large gravitationally bound states comprising of a scalar cloud containing exponentially large axion population numbers. The theory behind superradiance has been extensively covered in Ref. [5] with only the basics outlined below.

The condition for mode amplification of the scalar field requires the angular velocity of the $\mathrm{BH}$ horizon to exceed the angular phase velocity of the wave mode, defining the superradiance condition (see Fig. 4)

$$
\frac{\omega}{m}<\omega_{+},
$$

where $m$ is the spherical harmonic quantum number and $\omega_{+}$is the effective angular velocity of the $\mathrm{BH}$. The BHs dimensionless spin, $a_{*}$ is defined in the region $0 \leq\left|a_{*}\right|<1$ as,

$$
a_{*}=\frac{a}{r_{g}},
$$

in Boyer-Lindquist coordinates. The gravitational radius of the $\mathrm{BH}$ is,

$$
r_{g} \equiv G_{\mathrm{N}} M_{\mathrm{BH}}
$$

In parts of the following we shall work in units $c=\hbar=$ $G=1$ such that $r_{g} \equiv M_{\mathrm{BH}}$. The Kerr-Klein-Gordon system admits quasibound states with complex eigenfrequencies

$$
\omega_{n l m}=\omega_{R}+i \omega_{I},
$$

where $\left\{\omega_{R}, \omega_{I}\right\} \in \mathbb{R}$. The superradiance rates are defined as the small imaginary component of the energy of the free field solution on the Kerr background. Modes satisfying these conditions will grow exponentially over time identifying the presence of an instability in the Kerr spacetime. The orbitals around the $\mathrm{BH}$ are indexed by the overtone $(n)$, orbital multipole $(l)$ and azimuthal $(m)$ quantum numbers which satisfy $l \leq n-1$ and $|m| \leq l$ and form discrete sets, $\{n, l, m\}$ used to quantize the superradiant behavior. Superradiance requires evolving modes to corotate with the BH which satisfy, $m>0$. The dimensionless coupling of the gravitational $\mathrm{BH}$-scalar condensate system is,

$$
\alpha=r_{g} \mu_{\mathrm{ax}} \equiv \mu_{\mathrm{ax}} M_{\mathrm{BH}}
$$

in our choice of units. Figure 3 presents the coupling strength for potential regions of the axion mass parameter space open to investigation for $\mathrm{BH}$ masses spanning the stellar and supermassive limits.

The evolution of the axion field is defined by the characteristic eigenfrequencies corresponding to the instability time scales for the unstable modes of the system. The nature of scalar instabilities is well researched covering both the frequency [94-96] and time domains [97]. Analyzing the region of the parameter space where $\alpha \sim 1$, solutions for the unstable modes can be found using a numerical analysis of the wave equation $[96,98,99]$. When $\alpha$ surpasses unity WKB methods are formulated to evaluate the superradiance rate $[12,95]$.

It has been shown that it is possible to find analytical solutions to approximate the instability rate, incorporating matching techniques between different regimes of validity as a a function of $\alpha$. For a particular bound state if the superradiance condition is satisfied then providing that the instability rate is quicker than relevant astrophysical time scales, wave modes will extract energy and angular momentum from the $\mathrm{BH}$. It has been shown in the $\alpha \ll 1$ regime known as the "small mass approximation" that the evolution of the superradiant instability can be analytically described via a matched asymptotic expansion. This solution was initially derived by Detweiler to solve the Klein-Gordon equation of the scalar field perturbation [94]. The instability rate in the small mass approximation is defined as 


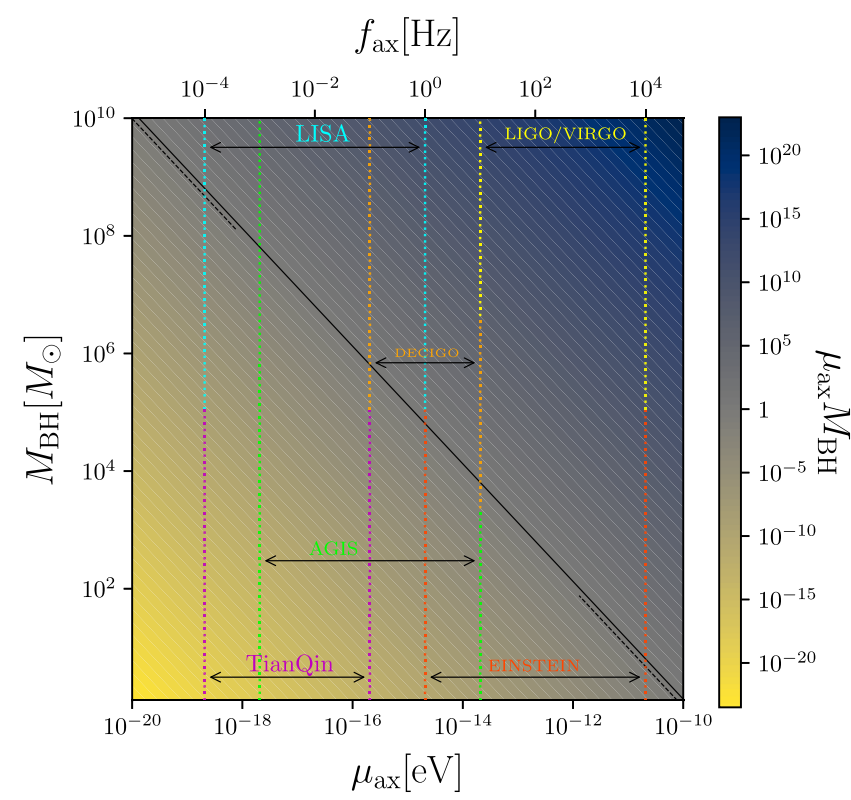

FIG. 3. The BH-scalar condensate coupling, $\alpha=\mu_{\mathrm{ax}} M_{\mathrm{BH}}$. The solid black line represents the unity limit for nonrelativistic and relativistic regimes. The dashed line corresponds to $\alpha=0.5$, the approximate limit in which the analytical approximation for the instability rate is valid. Dotted lines correspond to frequency ranges for monochromatic gravitational wave emission from the scalar cloud accessible to current and future GW observatories [87-93].

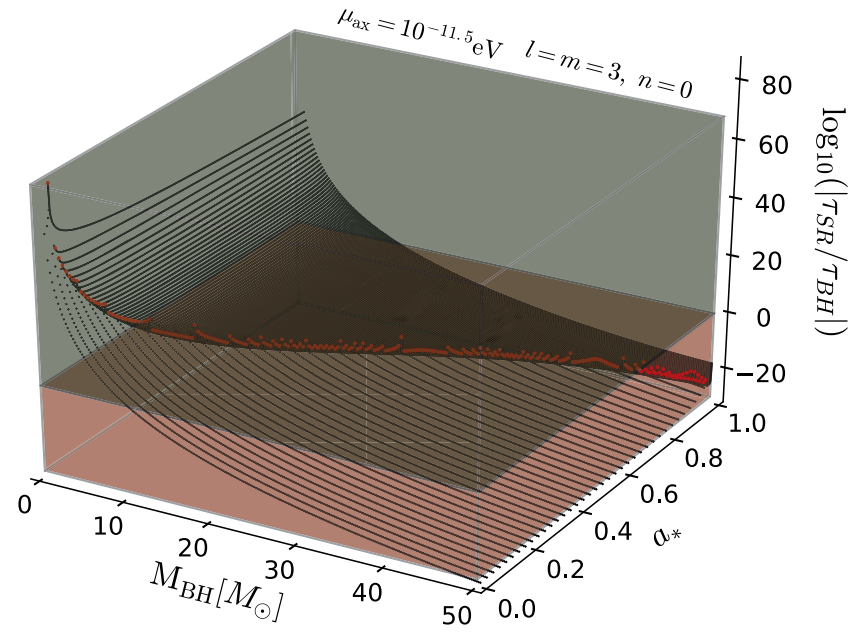

FIG. 4. Time scale ratios for the superradiance rates for an axion with mass $\mu_{\mathrm{ax}}=10^{-11.5} \mathrm{eV}$ compared with a typical $\mathrm{BH}$ astrophysical time scale, here taken to be $\tau_{\text {Salpeter }}[$ Eq. (21)]. Each cusp represents the analytical limit beyond which Eq. (4) is satisfied. The limit to the right of the cusp (sold line) represents the ratio defining the nature of the time scales where superradiance is apparent. The red volume defines the limit in the twodimensional $\mathrm{BH}$ mass/spin parameter space where superradiance occurs within the defined astrophysical time scale used to map the Regge plane isocontour limits.

$$
\Gamma_{n l m}=2 \mu_{\mathrm{ax}} r_{+}\left(m \Omega_{H}-\mu_{\mathrm{ax}}\right)\left(\mu_{\mathrm{ax}} M_{\mathrm{BH}}\right)^{4 l+4} \mathcal{C}_{n l m},
$$

where,

$$
\begin{aligned}
\mathcal{C}_{n l m}= & \frac{2^{4 l+2}(2 l+n+1) !}{n !(n+l+1)^{2 l+4}}\left[\frac{l !}{(2 l+1) !(2 l) !}\right]^{2} \\
& \times \prod_{j=1}^{l}\left[j^{2}\left(1-\frac{a^{2}}{M_{\mathrm{BH}}^{2}}\right)+4 r_{+}^{2}\left(\mu_{\mathrm{ax}}-m \Omega_{h}\right)^{2}\right]
\end{aligned}
$$

which is maximized close to the superradiance boundary. In Fig. 5 we present example superradiance rates under this formalism for a range of modes at fixed spin as a function of the axion/BH coupling, $\mu_{\mathrm{ax}} M_{\mathrm{BH}}$. The fastest growing mode occurs for $\Gamma_{011}$ when the spin is maximized, with the superradiance rates exponentially suppressed for higher values of $l$. The maximum superradiance rates are found by fixing the values of $l$ and $m$ such that, $l=m$ where $m$ determines the ability to satisfy the superradiance condition in Eq. (4). The value of $\Gamma_{n l m}$ has a limited dependence on the overtone mode, $n$. When the $\mathrm{BH}$ possesses significant spin higher-order overtone modes for larger values of $l=m$ can present greater superradiance rates as compared to the fundamental overtone mode. Analytically this is apparent for $l=m=4$ (see right panel of Fig. 5) where it has also been shown to occur for $l=m=3$ considering numerical solutions [100].

\section{Superradiant evolution for multiple fields}

Sequential to the formational phase of a $\mathrm{BH}$, superradiant evolution can begin via quantum fluctuations in the vacuum where each of the quantized superradiant levels begins to grow exponentially with their corresponding superradiance rates. The fastest-growing level which satisfies the superradiance condition always dominates the initial superradiant evolution until it has extracted enough spin so that the superradiance condition is no longer satisfied. Once the scalar cloud has extracted the maximal spin for the dominant mode the system can be considered as a (quasi)stationary hairy $\mathrm{BH}$ for astrophysical purposes. The $\mathrm{BH}$ energy loss through mass reduction is minimal compared to the shift in angular momentum due to the extent of the scalar cloud. Once the growth of the dominant level has stopped the $\mathrm{BH}$ will spend a significant portion of its lifetime on a Regge trajectory separating higher mode instability bounds. This can be seen from the basic intuition that as the higher modes of the $\mathrm{BH}$ begin to spin down the $\mathrm{BH}$ perturbing it from the Regge trajectory the negative component of the eigenfrequency for the previous mode dominates the evolution, spinning up the $\mathrm{BH}$. This process is apparent until a significant portion of the scalar density in the cloud is reduced from the previously dominant level. At this point the $\mathrm{BH}$ traverses the Regge plane towards the successive superradiant boundary, repeating the process until the time scales considered are too large for superradiance to occur. 


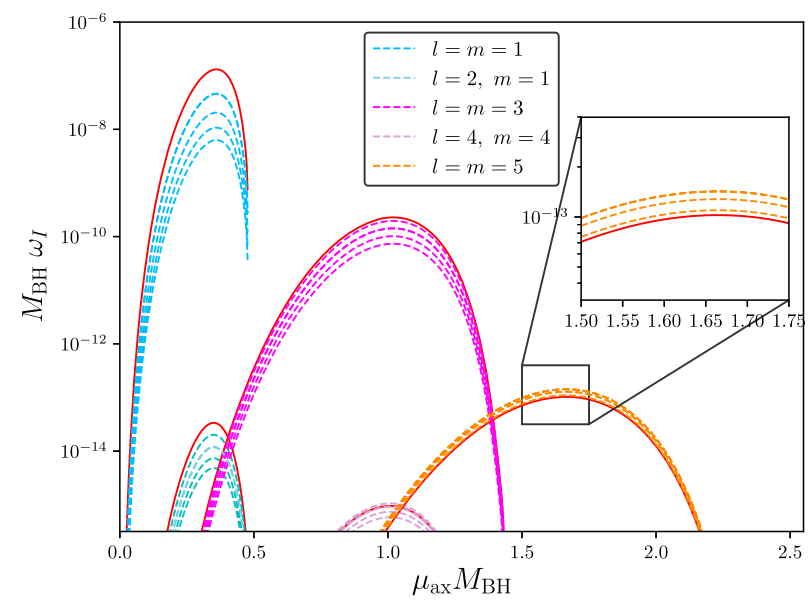

FIG. 5. Imaginary component of the bound-state frequency, $M_{\mathrm{BH}} \omega_{I}$ representing the superradiance instability rate, $\Gamma_{\mathrm{nlm}}$ as a function of the dimensionless coupling, $\alpha=\mu_{\mathrm{ax}} M_{\mathrm{BH}}$. Example superradiance rates are for the fundamental and higher-order overtone modes $n=0$ to 4 for configurations satisfying $l=m$ and $l>m$. The red lines correspond to the fundamental overtone modes, $n=0$ which become subdominant for values of $l=m \geq 4$.

If nonlinearities are taken into account level mixing can increase the time spent on the superradiance condition boundary via perturbations of the gravitational potential around the BH. Dissipation of the scalar cloud can occur through processes such as the annihilation of axions into gravitons or unbound axions [12,21]. In general the scalar cloud becomes maximally occupied before annihilation processes begin in the nonrelativistic limit. Further complications to the trajectory evolution of the $\mathrm{BH}$ could come from the bosenova phenomena, introducing intermediate stages comprising of bursts of GWs and phases spinning down the $\mathrm{BH}$ before the superradiance condition is finally saturated. Given the hierarchy of time scales between the superradiant instability and the GW emission from nonlinearities when compared to the dynamical time scale of the $\mathrm{BH}$ it is possible to study the system's evolution in the quasiadiabatic approximation for $N_{\mathrm{ax}}$ fields $[5,14,86]$. The total scalar energy flux from the superradiance process through the horizon is,

$$
\dot{E}=2 M_{S} \sum_{g=1}^{N_{\text {ax }}} \omega_{I, g}
$$

Disregarding accretion, the evolution of the system is described by the following equations:

$$
\begin{aligned}
& -\dot{E}_{S}=\dot{M}_{\mathrm{BH}}, \\
& -\dot{E}=\dot{M}_{\mathrm{BH}}+\dot{M}_{S},
\end{aligned}
$$

$$
\begin{aligned}
& -m \dot{E}_{S} / \sum_{g=1}^{N_{\mathrm{ax}}} \omega_{R, g}=\dot{J}_{\mathrm{BH}}, \\
& -m \dot{E} / \sum_{g=1}^{N_{\mathrm{ax}}} \omega_{R, g}=\dot{J}_{\mathrm{BH}}+\dot{J}_{S},
\end{aligned}
$$

where $E_{S}$ is the energy of the scalar cloud. The scalar cloud extracts mass and spin until reaching the saturation point. The final $\mathrm{BH}$ spin is,

$$
J_{\mathrm{BH}, \mathrm{F}}=\frac{4 m M_{\mathrm{BH}, \mathrm{F}}^{3} \sum_{g=1}^{N_{\mathrm{ax}}} \omega_{R, g}}{m^{2}+4 M_{\mathrm{BH}, \mathrm{F}}^{2} \sum_{g=1}^{N_{\mathrm{ax}}} \omega_{R, g}^{2}} .
$$

The final mass of the $\mathrm{BH}$ after the phase of superradiant evolution is defined by Eq. (14) were the variations in the defining $\mathrm{BH}$ parameters are related by,

$$
\delta J_{\mathrm{BH}}=\frac{m}{\sum_{g=1}^{N_{\mathrm{ax}}} \omega_{R, g}} \delta M_{\mathrm{BH}} .
$$

This defines the final mass of the $\mathrm{BH}$ :

$$
M_{\mathrm{BH}, \mathrm{F}}=M_{\mathrm{BH}, \mathrm{I}}-\frac{\sum_{g=1}^{N_{\mathrm{ax}}} \omega_{R, g}}{m}\left(J_{\mathrm{BH}, \mathrm{I}}-J_{\mathrm{BH}, \mathrm{F}}\right) .
$$

The true evolution of course is a complicated picture where nonlinearities must be accounted for along with the properties of each system. In particular for SMBHs their masses are generally accumulated via accretion which requires very significant perturbations in order to match the evolutionary traits a stellar $\mathrm{BH}$ may follow e.g., in terms of traversing the mass-spin Regge plane.

\section{The black hole mass-spin Regge plane}

A fundamental prediction stemming from superradiant instabilities of bosonic fields is the existence of exclusion regions in the BH Regge plane. Estimates of the instability time scale, $\tau_{\mathrm{SR}}$ partnered with reliable spin measurements for BHs, can be used to impose stringent constraints on the allowed masses of ultralight bosons. These bounds on the parameters of ultralight bosons follow from the requirement that in principle an astrophysical spinning BH should be stable over its lifetime. A superradiant instability time scale which acts faster than core processes such as accretion forms observational thresholds on the expected regions of the two-dimensional mass-spin parameter space BHs should fall in. Following the process of superradiant evolution a large number of $\mathrm{BH}$ observations should trace out the superradiance condition boundaries, mapping the Regge trajectories given the existence of as yet unidentified fields. For axions the shape of the gaps in the Regge plane 
are extremely sensitive to variations in the superradiant growth rate with the scalar mass. A BH therefore should be excluded from observational measurements given the existence of an ultralight boson if its spin is measured above the relevant level curves for different orbital states of the quantized modes for the field. The bounds for bosonic fields with spin are wider than those for axionlike particles and so the potentially large systematic errors in $\mathrm{BH}$ spin measurements could act as a current restriction to this approach for spin-0 fields. The axion mass window which can be probed is fixed by the heaviest supermassive BHs with accurate recorded spin measurements along with a lower bound defined by the lightest measured stellar mass BHs.

The current lower and upper bounds on $\mathrm{BH}$ masses from $\mathrm{x}$-ray spectroscopy and emission data covers the approximate region

$$
5 M_{\odot} \lesssim M_{\mathrm{BH}} \lesssim 5 \times 10^{8} M_{\odot},
$$

which defines the relevant axion mass window as,

$$
10^{-20} \mathrm{eV} \lesssim \mu_{\mathrm{ax}} \lesssim 10^{-11} \mathrm{eV}
$$

The isocontours defining the exclusion bounds are a function of the instability time scale and the boson mass. As the axion mass decreases the instability exclusion contours reduce in size. This corresponds to tighter instability regions which require larger spins for more massive BHs. Taking into account accretion and GW emissions can also slightly reduce the bounds in the Regge plane [86]. The time scales associated with the astrophysical processes of relevance change when considering different compact object systems.

For rapidly spinning $\mathrm{BH}$ candidates in x-ray binary systems or binary $\mathrm{BH}(\mathrm{BBH})$ mergers identified as detectable GW sources by LIGO more accurate constraints can be imposed when considering the typical time scales associated with a binary system's lifetime as other astrophysical processes such as accretion are subleading in this regard. A typical lower bound approximation for the lifetime of the binary system is given as $\tau_{\mathrm{BH}} \sim 10^{6} \mathrm{yrs}$ for the most accurate constraints. The most conservative limits come from exclusion regions constructed using the Hubble time $\tau_{\mathrm{H}} \sim 10^{10}$ yrs.

As opposed to stellar binary objects the relevant time scales for AGN in order for superradiance to maximally grow the scalar cloud for each quantized level come from accretion models. A statistical analysis of the exclusion limits over the whole BH mass region defined in Eq. (19) requires us to use a characteristic time scale derived from accretion considerations. The time scale for mass growth increases exponentially with an $e$-folding time given by a fraction $1 / f_{\text {Edd }}$ of the Salpeter time scale, where $f_{\text {Edd }}$ is the Eddington ratio for mass accretion. The accretion time scale is estimated using the Salpeter time for a $\mathrm{BH}$ radiating at its Eddington limit

$$
\tau_{\text {Salpeter }}=\frac{\sigma_{\mathrm{T}}}{4 \pi m_{\mathrm{P}}} \sim 4.5 \times 10^{7} \mathrm{yrs},
$$

where $\sigma_{\mathrm{T}}$ is the Thompson cross section and $m_{\mathrm{P}}$ is the proton mass [101]. In order to model the accretion time the following parameters can be introduced [14]:

$$
\tau_{\text {Salpeter }}=4.5 \times 10^{8} \mathrm{yrs} \frac{\eta}{f_{\text {Edd }}(1-\eta)},
$$

where $\eta$, the thin-disk radiative efficiency is a function of the spin related to a specific energy at the innermost stable circular orbit (ISCO). We select a typical value for the efficiency, $\eta=0.1$ and the most conservative value of $f_{\text {Edd }}=1$ to model the effects of accretion. This fixes the superradiant instability time scale as $\tau_{\mathrm{SR}}=45 \mathrm{Myrs}$. Increasing the bounds on $f_{\text {Edd }}$ allows for more optimistic models incorporating potential periods of super-Eddington accretion. A redefinition of $f_{\text {Edd }}$ holds the same equivalence as considering a subpopulation of degenerate mass fields (see Sec. IV B) or considering different astrophysical processes to define the superradiance time scale. Such considerations are a limitation in the "logistics" of encapsulating the behavior of the total BH spectrum and as such we follow the most conservative limit defined above.

An individual treatment of the instability time scales derived from the properties of the accretion disc stability for each $\mathrm{BH}$ candidate can be used to tighten constraints of the field mass exclusions [102]. As the time scale limits for the superradiant instability are increased the limits for each mode, $m$ will begin to saturate to the limits set by the boundaries of the superradiance condition. This effect is most prominent for higher-order modes on the spin axis of the Regge plane allowing for enhancements in the potential to constrain ultralight bosons using observations of $\mathrm{BHs}$ with spins that are a moderate fraction of the extremal limit.

Figure 6 details the exclusion bounds for the treatment of a single axion covering the full region of the axion mass window in Eq. (20), along with the full stellar BH and SMBH data presented in Table I. The primary axis displays the Regge exclusion bounds for an instability time scale $\tau_{\mathrm{SR}}=45 \mathrm{Myrs}$ as a function of the axion mass, $\mu_{\mathrm{ax}}$. The blue/orange data points are the stellar/SM BHs in Table I. The secondary axis displays the probability exclusion function formulated from the statistical model in Appendix A across the total $\mathrm{BH}$ mass range. The function "well" corresponds to the absence of any welldefined IMBH candidates. Well-defined mass and spin measurements for BHs covering the approximate region $10^{2} M_{\odot}-10^{6} M_{\odot}$ could fill the currently inaccessible portion of the parameter space and probe interesting masses for axions associated with GUT and supersymmetric 


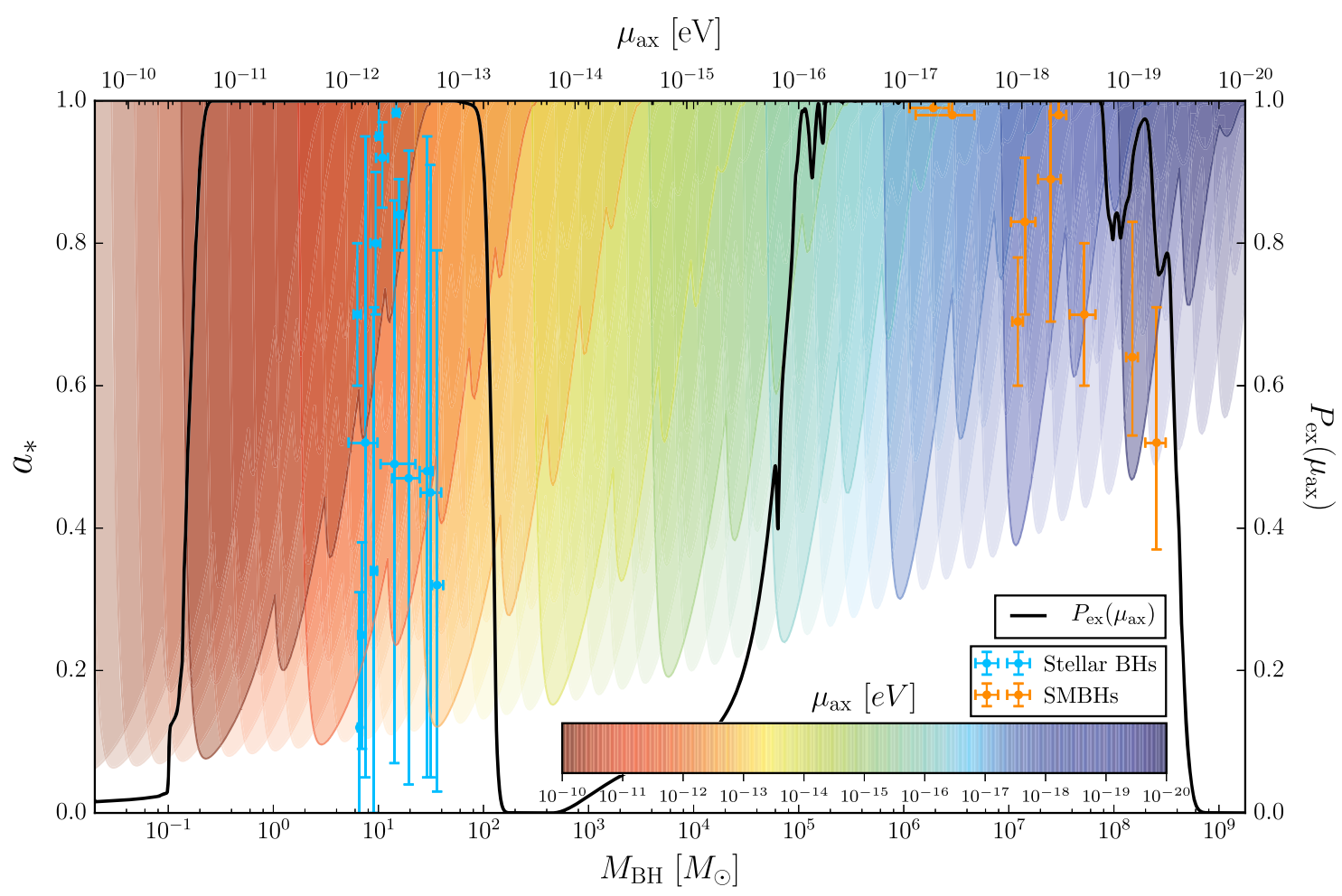

FIG. 6. Isocontour exclusion bounds with calculated total exclusion probabilities in the BH mass-spin Regge plane from superradiant instabilities with a single axion field with mass, $\mu_{\mathrm{ax}}$ spanning the limits in Eq. (20). The shaded regions represent instability thresholds shorter than the time scale $\tau_{\text {Salpeter }}$ in Eq. (21) for each value of the dominant orbital/azimuthal quantum numbers, $l=m=1$ to 5 . The blue data points are mass/spin estimates of stellar X-ray and BBH systems. The orange points correspond to mass/spin estimates of SMBHs from $\mathrm{X}$-ray reflection spectroscopy. The exclusion probability function (black line) is calculated using the statistical model in Appendix A using the BHs compiled in Table I and is given as a function of the axion mass spanning both the stellar and supermassive regimes.

models in string/M-theory. The most promising realization of detecting BHs in this space comes from the proposed space-based gravitational wave observatories such as the Laser Interferometer Space Antenna (LISA) (see Fig. 2).

\section{E. Black hole spin measurements from binary systems and active galactic nuclei}

The identification of compact systems has seen a steady increase over the past decades with a number of $\mathrm{x}$-ray binary sources and active galactic nuclei (AGN) now providing well-defined measurements for the masses and spins of these systems. Currently the main sources of error for catalogued BHs comes from the systematic errors when modeling the emission of the accreting disc of the system. Both stellar $\mathrm{BH}$ and $\mathrm{SMBH}$ measurements come from analyzing the $\mathrm{x}$-ray spectrum of the accretion disk for identified compact sources. Assuming that general relativity holds true as a valid description of the spacetime region outside the $\mathrm{BH}$ horizon and the ISCO of the accretion disk possesses a monotonic function potential then estimates on the spins of BHs can be made. In principle most $\mathrm{BH}$ candidates with well-defined parameter estimates come from either thermal continuum fitting of the inner accretion disk or inner disk reflection modeling in order to determine the size of the ISCO. In addition, BH spin data has recently been collected via the observations made of several BBH mergers by LIGO [27,103,104]. Currently such observations contain large errors on both the mass and spin of the BHs when compared to existing $\mathrm{x}$-ray binary system records. The resultant BHs formed from such astrophysical events cannot be included in considerations of constraining the masses of bosons given that their time scale for observation is less than typical instability time scales by definition in the process of identification. Generally though future generation ground-based detectors are still expected to produce large error measurements on BHs identified in this way and so impose a strong limitation on the accuracy of measurements used for constraints. Improvements in observatory sensitivity with space-based missions such as LISA [105] will open up the potential for a large catalogue of accurate $\mathrm{BH}$ measurements capable of probing a large potion of the cosmologically significant sector for axionlike fields. A large exclusion in the fully accessible space could also lead to tight constraints on how the axion population or subpopulations may be distributed when seeking realizations of desirable models in the context of cosmology. 
TABLE I. Stellar BH and SMBH systems used to apply constraints on axion masses and values of $N_{\mathrm{ax}}$ for various model mass spectra. BHs are selected with reliable mass and spin measurements and associated errors are quoted with their confidence limits and corresponding references. Stellar BH measurements come from both x-ray binary systems via x-ray continuum-fitting methods and BBH mergers from detected coalescence events at LIGO. SMBHs are measured AGN using x-ray reflection spectroscopy. Where two methods have been stated we use averaged posterior values for each. For review material and collections of stellar BHs see Refs. [22,23]. Compiled AGN data can be found in Refs. [24-26].

\begin{tabular}{|c|c|c|c|c|c|c|}
\hline Object & Method & Mass $\left(M_{\mathrm{BH}}\right)$ & $\operatorname{Spin}\left(a_{*}\right)$ & Mass CL & Spin CL & Ref. \\
\hline Stellar & & {$[M \odot]$} & & & & \\
\hline GW150914 (Primary) & EOBNR + IMRPhenom & $36.2_{-3.80}^{+5.20}$ & $0.32_{-0.29}^{+0.47}$ & $90 \%$ & $90 \%$ & [27] \\
\hline GW150914 (Secondary) & EOBNR + IMRPhenom & $29.1_{-4.40}^{+3.70}$ & $0.48_{-0.43}^{+0.47}$ & $90 \%$ & $90 \%$ & {$[27]$} \\
\hline GW151226 (Primary) & EOBNR + IMRPhenom & $14.2_{-3.70}^{+8.40}$ & $0.49_{-0.42}^{+0.43}$ & $90 \%$ & $90 \%$ & {$[27]$} \\
\hline GW151226 (Secondary) & EOBNR + IMRPhenom & $7.5_{-2.30}^{+2.30}$ & $0.52_{-0.47}^{+0.43}$ & $90 \%$ & $90 \%$ & {$[27]$} \\
\hline GW170104 (Primary) & Eff + Full precession & $31.2_{-6.00}^{+8.40}$ & $0.45_{-0.40}^{+0.46}$ & $90 \%$ & $90 \%$ & {$[28]$} \\
\hline GW170104 (Secondary) & Eff + Full precession & $19.4_{-5.90}^{+5.30}$ & $0.47_{-0.43}^{+0.46}$ & $90 \%$ & $90 \%$ & [28] \\
\hline Cygnus X-1 & Continuum (KERRBB2) & $14.8^{+1.00}$ & $\geq 0.983$ & $1 \sigma$ & $3 \sigma$ & {$[29] /[30]$} \\
\hline XTE J1550-564 & Continuum (KERRBB2) & $9.10_{-0.61}^{+0.01}$ & $0.34_{-0.34}^{+0.37}$ & $1 \sigma$ & $90 \%$ & {$[31] /[32]$} \\
\hline A $0620-00$ & Continuum (KERRBB2) & $6.61_{-0.25}^{+0.01}$ & $0.12_{-0.19}^{+0.19}$ & $1 \sigma$ & $1 \sigma$ & {$[33] /[34]$} \\
\hline $4 U 1543-475$ & Continuum (KERRBB) & $9.4_{-1.00}^{+1.00}$ & $0.8_{-010}^{+0.10}$ & $1 \sigma$ & $1 \sigma$ & {$[35] /[36]$} \\
\hline GRO J1655-40 & Continuum (KERRBB) & $6.30_{-0.50}^{+0.00}$ & $0.7_{-010}^{+0.10}$ & $95 \%$ & $1 \sigma$ & {$[37] /[38]$} \\
\hline GRS $1915+105$ & Continuum (KERRBB2) & $10.1_{-0.60}^{+0.50}$ & $\geq 0.95$ & $1 \sigma$ & $1 \sigma$ & {$[39] /[40]$} \\
\hline LMC X-1 & Continuum (KERRBB2) & $10.91_{-1.41}^{+1.41}$ & $0.92_{-0.07}^{+0.05}$ & $1 \sigma$ & $1 \sigma$ & {$[41] /[42]$} \\
\hline LMC X-3 & Continuum (KERRBB2) & $6.98_{-0.56}^{+0.56}$ & $0.25_{-0.16}^{+0.13}$ & $1 \sigma$ & $1 \sigma$ & {$[43] /[44]$} \\
\hline M33 X-7 & Continuum (KERRBB2) & $15.65_{-1.45}^{+1.45}$ & $0.84_{-0.05}^{+0.05}$ & $1 \sigma$ & $1 \sigma$ & {$[45] /[46]$} \\
\hline Supermassive & & $\times 10^{6}[M \odot]$ & & & & \\
\hline Mrk 335 & Reflection (Suzaku) & $14.20_{-3.70}^{+3.70}$ & $0.83_{-0.13}^{+0.09}$ & $1 \sigma$ & $90 \%$ & {$[47] /[48]$} \\
\hline Fairall 9 & Reflection (Suzaku) & $255.0_{-56.0}^{+56.0}$ & $0.52_{-0.15}^{+0.19}$ & $1 \sigma$ & $90 \%$ & {$[47] /[49]$} \\
\hline Mrk 79 & Reflection (Suzaku) & $52.40_{-14.40}^{+14.40}$ & $0.70_{-0.10}^{+0.10}$ & $1 \sigma$ & $90 \%$ & {$[47] /[50]$} \\
\hline NGC 3783 & Reflection (Suzaku) & $29.80_{-540}^{+5.40}$ & $\geq 0.98$ & $1 \sigma$ & $90 \%$ & {$[47] /[51]$} \\
\hline MCG-6-30-15 & Reflection (Suzaku) & $2.90_{-160}^{+1.80}$ & $\geq 0.98$ & $1 \sigma$ & $90 \%$ & {$[52] /[53]$} \\
\hline NGC 7469 & Reflection (Suzaku) & $12.20_{-1.40}^{+1.00}$ & $0.69_{-0.09}^{+0.09}$ & $1 \sigma$ & $90 \%$ & {$[47] /[54]$} \\
\hline Ark 120 & Reflection (Suzaku) & $150.0_{-19.0}^{+19.0}$ & $0.64_{-0.11}^{+0.19}$ & $1 \sigma$ & $90 \%$ & {$[47] /[48]$} \\
\hline Mrk 110 & Reflection (Suzaku) & $25.10_{-6.10}^{+6.10}$ & $\geq 0.89$ & $1 \sigma$ & $90 \%$ & {$[47] /[48]$} \\
\hline NGC 4051 & Reflection (Suzaku) & $1.91_{-0.78}^{+0.10}$ & $\geq 0.99$ & $1 \sigma$ & $90 \%$ & {$[47] /[55]$} \\
\hline
\end{tabular}

We restrict ourselves to considering only $\mathrm{BHs}$ with detailed mass and spin errors. Each BH chosen for our analysis therefore has upper and lower bounds on both their mass and spin with well-defined quoted uncertainties. In Table I we present all the stellar BHs and SMBHs used to constrain our axion distributions in Sec. IV along with their associated references. For a review of compiled stellar $\mathrm{BH}$ data see Refs. [22,106] and for SMBHs see Refs. [24,25].

\section{THE AXION MASS SPECTRUM}

The generic multiaxion Lagrangian is

$$
\mathcal{L}=-\sum_{i, j=1}^{N_{\mathrm{ax}}} \mathcal{K}_{i j} \partial_{\mu} \theta_{i} \partial^{\mu} \theta_{j}-\sum_{\alpha=1}^{n_{\text {inst }}} \sum_{j=1}^{N_{\mathrm{ax}}} \Lambda_{\alpha} U_{\alpha}\left(\mathcal{Q}_{j, \alpha} \theta_{j}+\delta_{\alpha}\right)
$$

where $\theta_{i}$ are the dimensionless axion fields, $\mathcal{K}_{i j}$ is the kinetic matrix with mass dimension two, and $U$ is a general periodic instanton potential with charge matrix $\mathcal{Q}$ and phases, $\delta$. By expanding the potential to the mass term only and diagonalizing, Eq. (23) can be reduced to the simple form:

$$
\mathcal{L}=-\frac{1}{2} \partial_{\mu} \phi_{i} \partial^{\mu} \phi_{i}-\frac{1}{2} \operatorname{diag}\left(\mu_{\mathrm{ax}}^{2}\right) \phi_{i} \phi_{i}
$$

The spectrum of the model is given by the mass eigenvalues, $\left\{\mu_{i}\right\}$, which can be determined after expanding the instanton potential to quadratic order and obtaining a mass matrix, $\mathcal{M}_{i j}$. Diagonalizing these matrices following the methodology in Appendix B 1 gives the mass eigenstates of a spectrum of fields. The canonically normalized dimensionful mass eigenstate fields, $\phi_{i}$, are defined in Eq. (B7) from the eigenvalues of the kinetic matrix. Adopting random matrix models for $\mathcal{K}_{i j}$ and $\mathcal{M}_{i j}$ it 

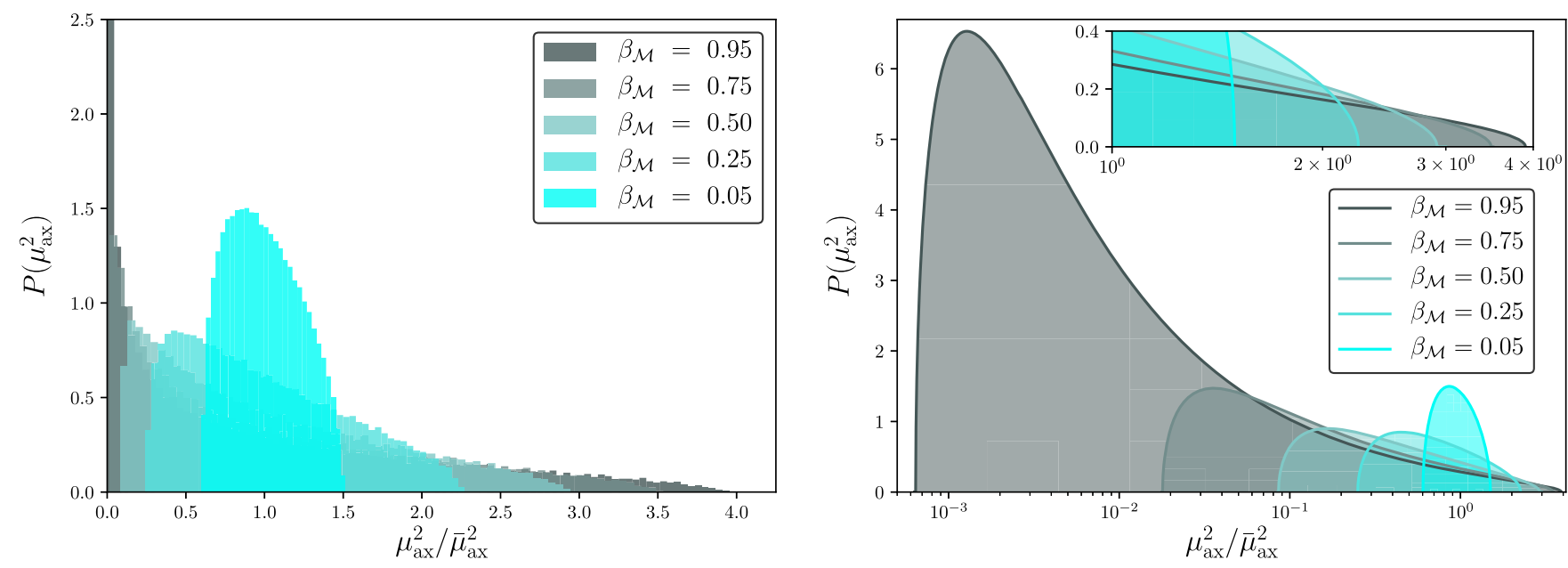

FIG. 7. Marčhenko-Pastur model normalized eigenvalue spectra and probability density functions for axion masses, $\mu_{\mathrm{ax}}^{2}$ with linear and logarithmic scales respectively. Each panel represents five selected values of the spectrum-shaping parameter $\beta_{\mathcal{M}}$ approximately covering its defining interval $\beta_{\mathcal{M}} \in(0,1]$. Left panel: The mass distribution converges to the Marčhenko-Pastur limiting law as $N_{\mathrm{ax}} \rightarrow \infty$. Asymptotically the largest eigenvalue fluctuations outside its defined compact interval are determined by the Tracy-Widom law [107]. Right panel: Probability density functions for each of the associated distributions in the left panel displayed on a logarithmic mass scale. Inset: As $\beta_{\mathcal{M}}$ increases the positive logarithmic displacement of the upper bound [Eq. (27)] is limited compared to the negative displacement of the lower bound [Eq. (28)] away from the mean scale of the distribution, $\bar{\mu}_{\mathrm{ax}}^{2}$.

is possible to determine the distribution of $\left\{\mu_{i}\right\}$ for various models. This process is reviewed in Appendix B and covered extensively in Ref. [65]. In the following we will consider just two simple models for the mass eigenvalues.

The first follows the celebrated Marčhenko-Pastur law for the eigenvalues of white Wishart matrices [80]. The spectrum is thought to describe type-IIB string theory models of inflation with large numbers of axions [81]. The limiting distribution as the matrix size goes to infinity is given by

$$
P\left(\mu_{\mathrm{ax}}^{2}\right)=\left\{\begin{array}{l}
\frac{1}{2 \pi \mu_{\mathrm{ax}}^{2} \beta_{\mathcal{M}} \bar{\mu}_{\mathrm{ax}}^{2}} \sqrt{\left(\gamma_{+}-\mu_{\mathrm{ax}}^{2}\right)\left(\mu_{\mathrm{ax}}^{2}-\gamma_{-}\right)}, \\
0,
\end{array}\right.
$$

on the compact interval

$$
\gamma_{-} \leq \mu_{\mathrm{ax}}^{2} \leq \gamma_{+},
$$

where $\gamma_{+}$and $\gamma_{-}$are defined as,

$$
\begin{aligned}
& \gamma_{+}=\bar{\mu}_{\mathrm{ax}}^{2}\left(1+\sqrt{\beta_{\mathcal{M}}}\right)^{2}, \\
& \gamma_{-}=\bar{\mu}_{\mathrm{ax}}^{2}\left(1-\sqrt{\beta_{\mathcal{M}}}\right)^{2} .
\end{aligned}
$$

The expectation value of $\mu_{\mathrm{ax}}^{2}$ is $\bar{\mu}_{\mathrm{ax}}^{2}$ and the shape parameter $0<\beta_{\mathcal{M}} \leq 1$ determines the spread of the distribution, with large $\beta_{\mathcal{M}}$ giving larger spreads as shown in the right panel of Fig. 7. The distribution for random realizations with finite $N_{\mathrm{ax}}$ is shown in the left panel of Fig. 7, and is well fit by the limiting law.
Our second model for the mass eigenvalues follows from the "M-theory axiverse" [64]. In this case the mass eigenvalues follow an approximately log-normal distribution [65], as shown in Fig. 8. ${ }^{1}$ In this example, the spread is controlled by the shaping parameter $\beta_{\mathcal{M}}=N_{\mathrm{ax}} / N_{\text {inst }}$ which takes values $0<\beta_{\mathcal{M}} \leq 1$. Increasing $\beta_{\mathcal{M}}$ leads to a larger mean and smaller variance.

There are five parameters in the model of Ref. [65] in total, but here we use a two-parameter approximate fit:

$$
P\left(\mu_{\mathrm{ax}}^{2}\right)=\frac{1}{\sqrt{2 \pi \sigma^{2}}} \exp \left[\frac{-\log _{10}\left(\mu_{\mathrm{ax}} / \bar{\mu}_{\mathrm{ax}}\right)^{2}}{2 \sigma^{2}}\right] .
$$

The mean of the log-normal distribution can be related to the expectation value of the 3-cycle volumes in the $G_{2}$ manifold, $\left\langle V_{X}\right\rangle$ [see Eq. (B21)], and as in the above example, the variance, $\sigma^{2}$, can be controlled by the number of instantons in the potential sum. The variance of the lognormal distribution is dimensionless, and so should take on some $\mathcal{O}(1)$ value. In Ref. [65] we typically found $\sigma \gg 1$.

Axion self-interactions can also play an important role in $\mathrm{BH}$ superradiance. In principle, by expanding the instanton potential to higher orders our RMT approach could lead to a distribution for the quartic interaction tensor:

$$
\mathcal{L}_{\text {int }}=\lambda_{i j k l} \phi_{i} \phi_{j} \phi_{k} \phi_{l}
$$

\footnotetext{
${ }^{1}$ The naive expectation of log-flat eigenvalues turns out not to be realized for large numbers of fields after applying rotations to the canonical basis.
} 


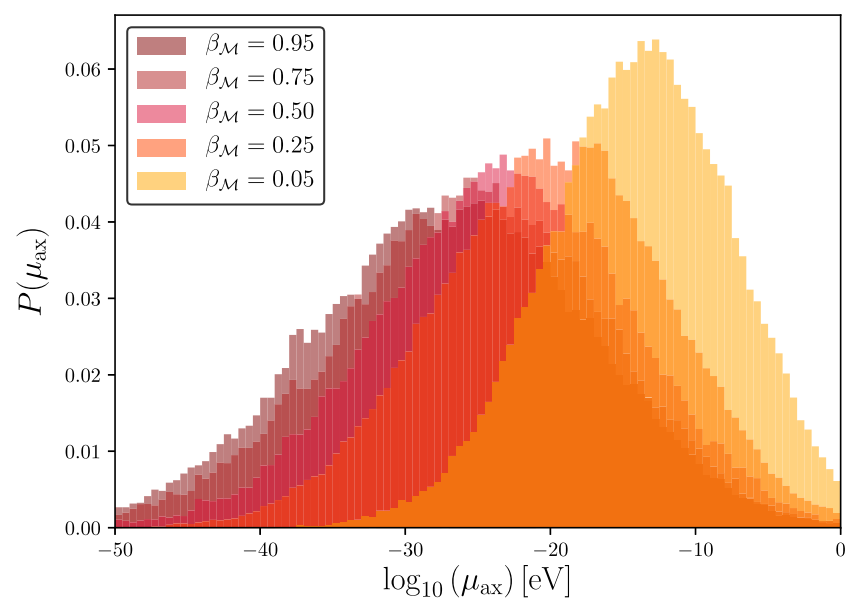

FIG. 8. M-theory model eigenvalue spectra for axion masses $\mu_{\mathrm{ax}}$ for different values of the spectrum-shaping parameter $\beta_{\mathcal{M}}$. The mass spectra converge to an approximate log-normal distribution in the mass eigenstate basis. Each spectrum is constructed using a fixed value of the average three-cycle volume, $\left\langle V_{X}\right\rangle=25$ required for GUT scale unification.

We are unaware of any study of the distribution of $\lambda_{i j k l}$ in RMT, and thus the treatment of interactions is beyond the scope of the present work. For sparse charge matrices the flavor-changing, nondiagonal, entries in $\lambda_{i j k l}$ will be rare.

If the attractive self-interactions are too strong then the superradiant cloud collapses via a bosenova before it can extract large amounts of spin from the BH. Superradiance can also be shut off by nonlinear level mixing, or affected by axion emission due to annihilations [12,17,21]. The interaction tensor can used to calculate these rates, e.g., for axion emission via the $\phi \phi \phi \rightarrow \phi$ process. The level mixing will be enhanced if the $\lambda_{i j k l}$ are nondiagonal and allow scattering of axions of different flavors. Decays from one flavor into another will have a similar effect of additional cooling of the cloud as the axion-photon coupling considered in Ref. [12]. The bosenova critical size, $N_{\text {bosenova }}$, could also become smaller in such a case due to the increased phase space for the scattering. How these and other nonlinear effects compete with the basic increase of the $\mathrm{BH}$ superradiance rate and increased probability of mass outliers at large $N_{\mathrm{ax}}$ is unclear.

Using the single instanton, dilute gas potential, $V(\phi)=$ $\mu^{2} f_{a}^{2}\left[1-\cos \left(\phi / f_{a}\right)\right]$ for a single field, it can be shown that the ratio of emission via the quartic interaction compared to graviton emission due to annihilations is given by [12]

$$
\frac{P_{\lambda}}{P_{\text {grav }}} \approx 10^{-2} \alpha^{4} \frac{M_{a}}{M_{\mathrm{BH}}}\left(\frac{M_{p l}}{f_{a}}\right)^{4} .
$$

The overall strength of the interactions, and their importance relative to gravity, is controlled by the axion decay constants, $f_{a}$. The $f_{a}$ distributions for multiple fields derived from RMT can be computed (see e.g., Ref. [65]). Distributions with a high probability of small decay constants will have nonlinearities dominated by selfinteractions, while for those with a high probability of large decay constants the pure-gravity results can be used. Since we consider BH superradiance dominated by gravity, our results should be understood to apply strictly to distributions dominated by large $f_{a}$. Taking the singlefield results of Ref. [21] as a guide, this should be for $f_{a} \gtrsim 10^{14-16} \mathrm{GeV}$. In the context of string models, our results should apply well to small-volume compactifications [108], whereas self-interactions will play an important role in the large-volume scenario [68].

\section{RESULTS}

\section{A. Single field}

In this short section, we begin the presentation of our results by computing single-field limits to check that our methodology is consistent with other results in the literature. Our statistical methods are described in Appendix A, and we calculate the exclusion probability, $P_{\mathrm{ex}}\left(\mu_{\mathrm{ax}}\right)$.

Treating the stellar BHs and SMBHs as a single data set, our results for a single axion field with mass $\mu_{\mathrm{ax}}$ are shown in Fig. 6, superimposed on the Regge plane with the data. In this combined data set the exclusion probability remains finite over a range of intermediate axion masses due to the large mass errors on the lightest SMBHs. The absence of IMBHs means that the regions with $P_{\mathrm{ex}}\left(\mu_{\mathrm{ax}}\right)>0.68$ (" $1 \sigma$ exclusion") do not overlap between the two data sets and they can be considered separately.

The exclusion probability for the stellar BH data set is shown in the right panel of Fig. 9. The high quality of these measurements, and the large number of them, leads to a smooth exclusion probability. At the $95 \%$ C.L. the stellar BHs exclude

$$
7 \times 10^{-14} \mathrm{eV}<\mu_{\mathrm{ax}}<2 \times 10^{-11} \mathrm{eV} .
$$

The exclusion probability for the SMBH data set is shown in the left panel of Fig. 9. The data is of generally poorer quality than the stellar data, with certain systems containing significantly large mass errors. It is also much sparser, with fewer SMBHs in the set. The sparseness of the data leads to oscillatory features in the exclusion probability, driven by the shape of the BH superradiance contours for each of the modes, with the exclusions being driven by individual BHs. This causes the probability of exclusion to oscillate between the $95 \%$ C.L. region and the $68 \%$ C.L. region when transitioning between certain BHs (faded red lines in the left panel of Fig. 9). The largest candidate, Fairall 9 drives the nonmonotonic nature of the function at low axion masses. The large mass errors lead to nonzero exclusion probability extending to large axion masses. Taking the outer edge of the $95 \%$ C.L. region, the SMBHs exclude 

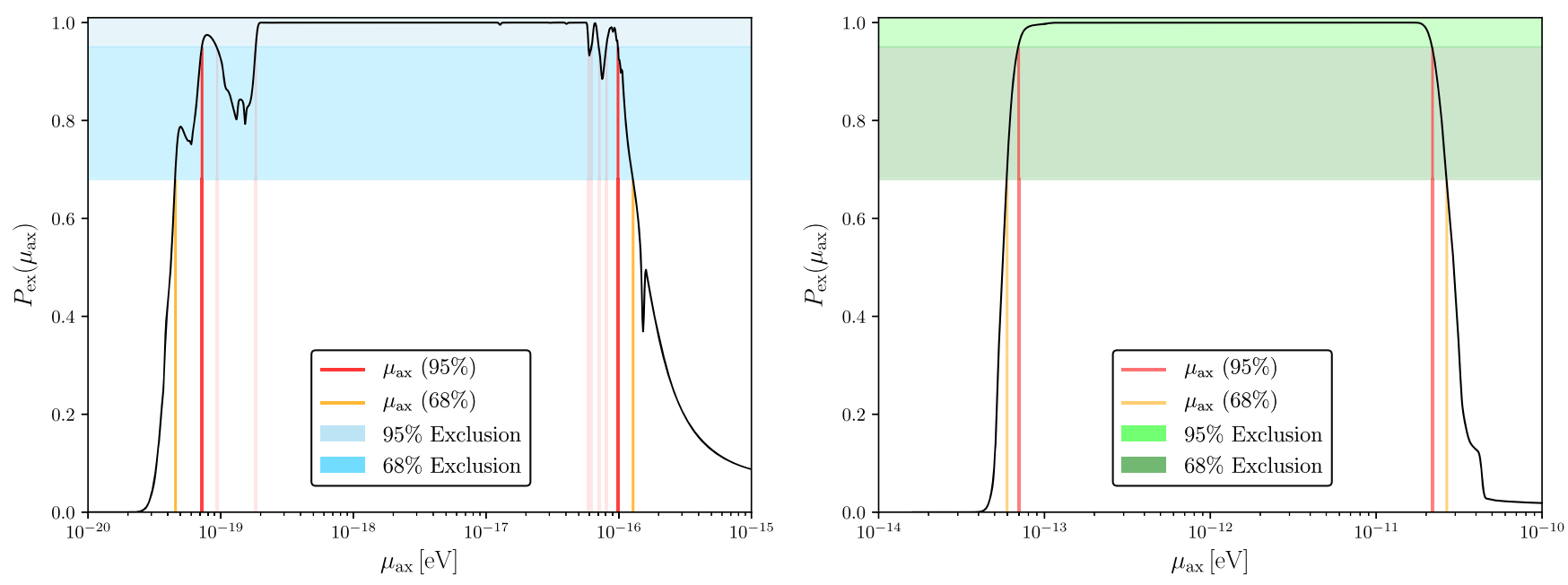

FIG. 9. Constraints on the masses of ultralight axions, $\mu_{\mathrm{ax}}$ for singular fields determined by the total probability of exclusion calculated using the methodology in Appendix A via Eq. (A1). Exclusion bounds are presented in the $68 \%$ and $95 \%$ confidence intervals as a function of $\mu_{\mathrm{ax}}$ with orange/red lines representing the upper and lower limits of the $68 \% / 95 \%$ interval. Left panel: Limits determined using the SMBHs given in Table I. Right panel: Limits determined using stellar mass BHs given in Table I.

$$
7 \times 10^{-20} \mathrm{eV}<\mu_{\mathrm{ax}}<1 \times 10^{-16} \mathrm{eV}
$$

Our exclusions for the stellar BH and SMBH data sets are consistent with the results of Refs. [21,102], after accounting for the differences in the data sets and methodology used. In particular comparing to Ref. [21] our choice to include $\mathrm{BBH}$ coalescence events with large masses when partnered with their large uncertainties push the constraints to incorporate lower masses, increasing the lower bound on the axion mass exclusion.

\section{B. Degenerate masses}

We now begin to consider cases with multiple axion masses. Treating the degenerate case is trivial for any number of axions $N_{\mathrm{ax}}$ with identical masses, $\mu_{\mathrm{ax}}$. Since the rate is additive in $N_{\text {ax }}$ we have

$$
\Gamma_{\text {tot }}=N_{\mathrm{ax}} \Gamma
$$

Therefore, setting $\tau_{\mathrm{BH}} \Gamma_{\text {tot }}=1$ is equivalent to the singlefield case with the time scale rescaled as $\tau_{N}=N_{\mathrm{ax}} \tau_{\mathrm{BH}}$. Thus, for the degenerate case the exclusion probabilities are trivial to compute for any $N_{\mathrm{ax}}$, and they will simply grow wider for increasing $N_{\mathrm{ax}}$ corresponding to larger rates. In Fig. 10 we show the effect on the Regge plane with a degenerate population of axions with masses $\mu_{\mathrm{ax}}=10^{-12.75} \mathrm{eV}$. It is clear that an increase in $N_{\mathrm{ax}}$ can lead to an exclusion on $\mu_{\mathrm{ax}}$ where there was not one in the single-field case (purple limits). As the instability thresholds sweep through the Regge plane as a function of the axion mass, the wider instability limits possess the ability to "catch" lighter BHs in their exclusion bounds.
We present the exclusion probabilities $P_{\mathrm{ex}}\left(\mu_{\mathrm{ax}}\right)$ for various values of $\log _{10} N_{\mathrm{ax}}$ for each regime in the left and right panels of Fig. 11. The contours in Fig. 10 always increase in the direction of smaller $M_{\mathrm{BH}}$, and so the constraints in Fig. 11 only broaden relative to the singlefield case for smaller axion masses. For SMBHs, where the higher harmonics play a role in the exclusion, the oscillations in the exclusion probability at high mass are also mildly affected. This is shown in the inset of the left panel of Fig. 11. Extremely large values of $N_{\text {ax }}$ quench the oscillations from the instability bounds of the higher-order modes, saturating the upper bounds on the constraints.

The 95\% excluded regions for $\mu_{\mathrm{ax}}$ for the degenerate case change by less than an order of magnitude compared to the single-field case for $N_{\mathrm{ax}} \lesssim 10^{5}$. This shows that the increase in the superradiance rate for multiple fields [i.e., the rate sum in Eq. (3)] can be virtually neglected when computing the exclusion probability, even in the most extreme case of a very large number of degenerate superradiant fields.

\section{Mass distributions}

There are two effects on $\mathrm{BH}$ superradiance constraints for mass distributions. The first is the effect of rate addition, and the second is the effect of an overlap between the mass distribution and the exclusion probability. The results of the previous section show that even for the extreme case of degenerate masses, this effect is virtually negligible in the exclusion probability for $\mu_{\mathrm{ax}}$. Rate addition will be even more negligible for mass distributions with finite width, where off-resonant superradiance rates are exponentially suppressed. This leaves probability overlap as the dominant effect for mass distributions of finite width. With the effect of rate addition neglected, the exclusion probability for a 


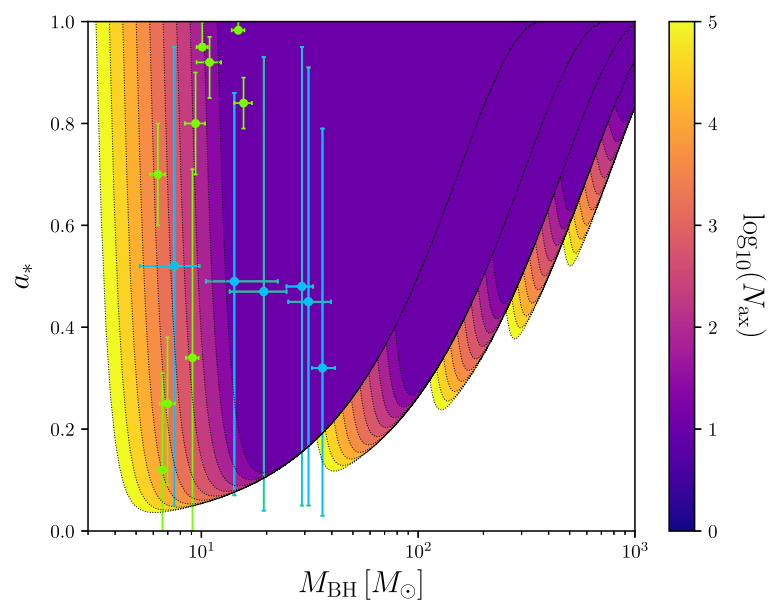

FIG. 10. Isocontour exclusion regions for degenerate mass axion populations with $N_{\mathrm{ax}}=\mathcal{O}(1) \rightarrow \mathcal{O}\left(10^{5}\right)$ in the stellar BH parameter space. The limits for the instability threshold are obtained by fixing the superradiant instability time scales for each value of the orbital/azimuthal quantum numbers, $1=\mathrm{m}=1$ to 5 equal to $\tau_{\text {Salpeter }}$ [Eq. (21)] for an axion mass $\mu_{\mathrm{ax}}=10^{-12.75} \mathrm{eV}$. Large values of $N_{\mathrm{ax}}$ effectively correspond to greater superradiance instability time scales considering a single field. Green data points are mass/spin estimates of x-ray binary stellar BH candidates. Blue data points are primary and secondary sources from $\mathrm{BBH}$ coalescence detections at LIGO.

mass distribution is trivial to construct from the exclusion probability for a single mass from the overlap integral. We use the probability that a model is allowed, since this trivially accounts for the combinatorics, and the excluded probability is in turn found trivially from this. Let $P_{\mathrm{al}}\left(\mu_{\mathrm{ax}} \mid N_{\mathrm{ax}}=1\right)=1-P_{\mathrm{ex}}\left(\mu_{\mathrm{ax}} \mid N_{\mathrm{ax}}=1\right)$ be the probability that a given axion mass is allowed, assuming just one axion field. We then have that in a given model $\mathcal{M}$ with one axion, the probability that some parameters $\theta$ are allowed is

$P_{\mathrm{al}}\left(\theta, N_{\mathrm{ax}}=1 \mid \mathcal{M}\right)=\int \mathrm{d} \mu_{\mathrm{ax}} p\left(\mu_{\mathrm{ax}} \mid \theta, \mathcal{M}\right) P_{\mathrm{al}}\left(\mu_{\mathrm{ax}} \mid N_{\mathrm{ax}}=1\right)$,

where $\mathrm{d} \mu_{\mathrm{ax}} p\left(\mu_{\mathrm{ax}} \mid \theta, \mathcal{M}\right)$ is the probability distribution for $\mu_{\mathrm{ax}}$ in the model. The single axion allowed regions were evaluated numerically in Sec. IVA and the integral in Eq. (35) can be evaluated numerically given $p\left(\mu_{\mathrm{ax}} \mid \theta, \mathcal{M}\right)$. The above trivially generalizes to the case of $N_{\mathrm{ax}}$ fields:

$P_{\mathrm{al}}\left(\theta, N_{\mathrm{ax}} \mid \mathcal{M}\right)=\left[\int \mathrm{d} \mu_{\mathrm{ax}} p\left(\mu_{\mathrm{ax}} \mid \theta, \mathcal{M}\right) P_{\mathrm{al}}\left(\mu_{\mathrm{ax}} \mid N_{\mathrm{ax}}=1\right)\right]^{N_{\mathrm{ax}}}$.

The exclusion probability for $N_{\text {ax }}$ fields is then given by $P_{\mathrm{ex}}\left(\theta, N_{\mathrm{ax}} \mid \mathcal{M}\right)=1-P_{\mathrm{al}}\left(\theta, N_{\mathrm{ax}} \mid \mathcal{M}\right)$.

\section{The Marčhenko-Pastur distribution}

The Marčhenko-Pastur distribution depends on two parameters: a mean mass, $\bar{\mu}_{\mathrm{ax}}$, and a shape parameter, $\beta_{\mathcal{M}}$. In order to probe the potential of a spectrum of fields scanning the Regge plane analogous to our single-field constraints we highlight several interesting configurations. Consider the case $\bar{\mu}_{\mathrm{ax}}=10^{-13} \mathrm{eV}$, shown in the left panel of Fig. 12. A single axion at this mass is excluded by the stellar $\mathrm{BH}$ data. However, for large spreads, i.e., $\beta_{\mathcal{M}} \rightarrow 1$, the mode of the distribution moves to smaller values of the mass (shown in the right panel of Fig. 7), which are not
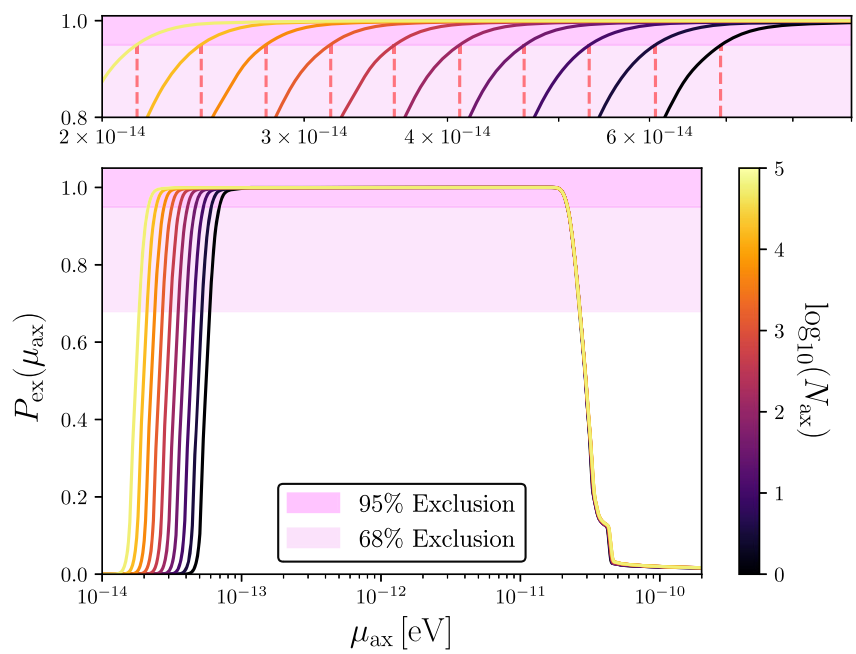

FIG. 11. Constraints on masses of ultralight axions, $\mu_{\mathrm{ax}}$, via the total exclusion probability in the $68 \%$ and $95 \%$ confidence limits for large numbers, $N_{\mathrm{ax}}$, of degenerate fields. Upper panels: Dashed red lines represent the shift of the lower bound in the $95 \%$ confidence limit, which decreases as $N_{\mathrm{ax}}$ increases. Left panel: Exclusion probability for the SMBH data set. Inset: Oscillatory behavior of the exclusion probability due to higher values of the orbital/azimuthal quantum numbers passing over low-mass SMBHs. Right panel: Exclusion probability for the stellar BH data set. 

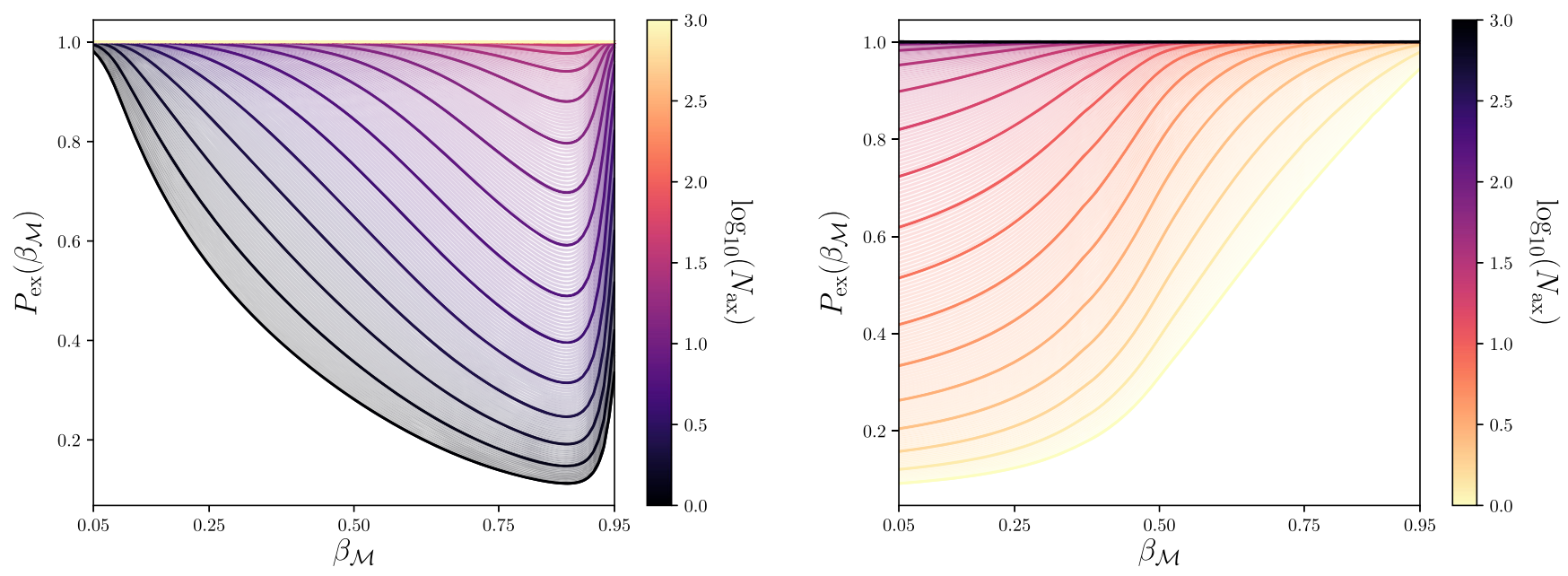

FIG. 12. Probability of exclusion as a function of the dimensionless shaping parameter, $\beta_{\mathcal{M}}$ defining the mass spectra in the Marčhenko-Pastur model for $N_{\mathrm{ax}}=1 \rightarrow 1000$. In general large populations or subpopulations are heavily constrained as the field masses saturate the limiting spectrum of the model. Left panel: The mean of the distribution is fixed to $\bar{\mu}_{\mathrm{ax}}=10^{-13} \mathrm{eV}$ on the edge of the constrained region from stellar BHs. Right panel: The mean of the distribution is fixed to $\bar{\mu}_{\mathrm{ax}}=10^{-15} \mathrm{eV}$ inside the "well" of the constrained region from both stellar BHs and SMBHs.

constrained. Eventually at still larger $\beta_{\mathcal{M}}$ the mode moves down to masses excluded by the SMBH data. In Fig. 13 we present the axion mass window open to superradiance for various distribution mean scales $\bar{\mu}_{\text {ax }}$. Increasing $N_{\text {ax }}$ makes the exclusion probability grow, and for all $\beta_{\mathcal{M}}$ there is a maximum $N_{\mathrm{ax}} \approx 20$ above which the model is excluded at better than the $95 \%$ C.L for all $\beta_{\mathcal{M}}$ (see Fig. 13). The maximum $N_{\text {ax }}$ allowed grows with $\beta_{\mathcal{M}}$.

Now consider the case $\bar{\mu}_{\mathrm{ax}}=10^{-15} \mathrm{eV}$, shown in the right panel of Fig. 12. In this case, the mean mass is in

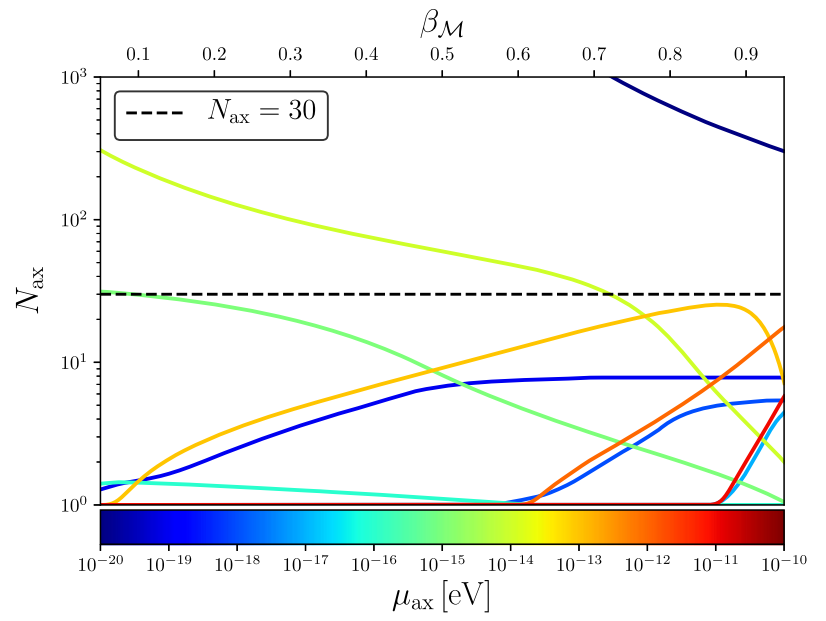

FIG. 13. Contours representing the 95\% exclusion for Marčhenko-Pastur axion mass distributions as a function of the distribution shape, $\beta_{\mathcal{M}}$, and number of fields, $N_{\mathrm{ax}}$, for various distribution mean scales, $\bar{\mu}_{\mathrm{ax}}$. Regions above the contours are excluded. Large numbers of fields are constrained for a significant region of the probable axion mass space, with $N_{\text {ax }} \geq 30$ constrained for a wide range of $\beta_{\mathcal{M}}$ over the considered scales. between the stellar and SMBH exclusions, and is allowed by the data. Thus, increasing $\beta_{\mathcal{M}}$ now increases the exclusion probability. The nonzero exclusion probability at $\mu_{\mathrm{ax}}=10^{-15} \mathrm{eV}$ coming from the lowest-mass points of the SMBH data with large error causes the exclusion probability to grow as $N_{\text {ax }}$ increases even for small $\beta_{\mathcal{M}}$. Once again, there is a maximum $N_{\mathrm{ax}} \approx 50$ above which the model is excluded at better than the 95\% C.L for all $\beta_{\mathcal{M}}$ (see Fig. 13). The maximum $N_{\text {ax }}$ allowed decreases with $\beta_{\mathcal{M}}$.

Motivated by the peak in the Calabi-Yau distribution along the self-mirror manifold line, Fig. 14 shows constraints on $\bar{\mu}_{\text {ax }}$ at fixed $\beta_{\mathcal{M}}=0.5$. The excluded region has the same approximate shape as the single-field exclusions for small $N_{\text {ax }}$. As $N_{\text {ax }} \rightarrow 1$ the exclusion limits trace out the constraints for the single-field case up to statistical fluctuations about the mean scale. The softer edges of the untouched regions when compared with the single-field exclusion bounds come from the nonequidistant logarithmic spread of the mass spectrum about the mean scale when $\beta_{\mathcal{M}}=0.5$. Reducing $\beta_{\mathcal{M}}$ relaxes the limits to fully match the single-field case in the low- $N_{\text {ax }}$ limit. Increasing the number of fields, the model is excluded at better than the 95\% C.L. for the range of mean masses shown for all $N_{\text {ax }} \gtrsim 100$.

\section{The M-theory axiverse: The $Q C D$ axion, GUTs, and fuzzy DM}

The axion mass spectrum of the M-theory axiverse [64] was computed from RMT models in Ref. [65] and is well described by a log-normal distribution. The $95 \%$ excluded region in $\left(\sigma, N_{\mathrm{ax}}\right)$ for the log-normal distribution across a range of central values is shown in Fig. 1. We now derive 


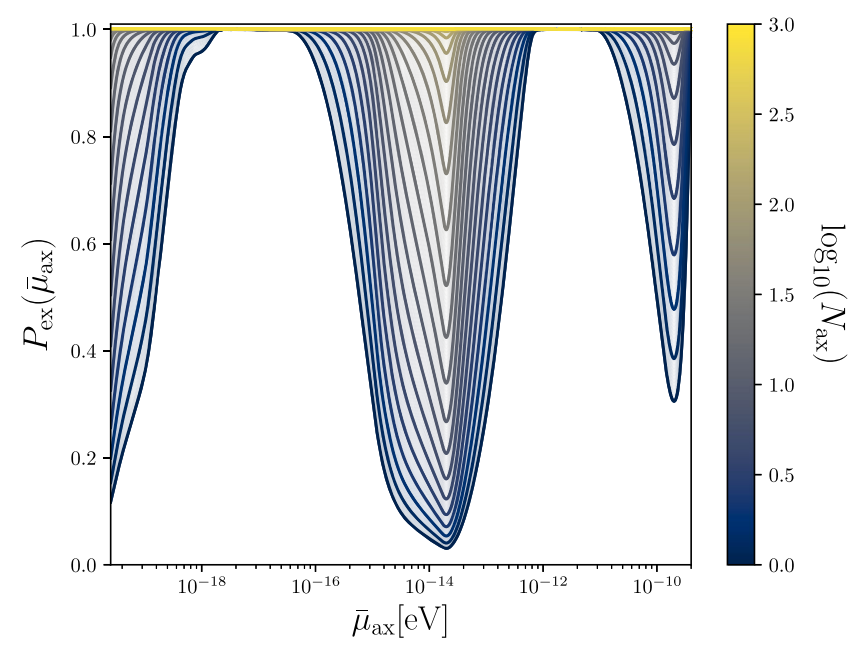

FIG. 14. Probability of exclusion as a function of the Marčhenko-Pastur distribution mean scale, $\bar{\mu}_{\mathrm{ax}}$ for $N_{\mathrm{ax}}=1 \rightarrow 1000$. Each probability function is determined using a fixed shape parameter, $\beta_{\mathcal{M}}=0.5$. In general large populations or subpopulations are heavily constrained as the field masses saturate the limiting spectrum of the model. The singular field bounds trace the limits in Fig. 6 defining the white region within statistical fluctuations of the mean.

BH superradiance constraints on three scenarios of interest realized approximately from this simple model for the M-theory axiverse. The M-theory axiverse with GUT scale unification predicts the existence of an axion with

$$
\mu_{\mathrm{GUT}} \approx 10^{-15} \mathrm{eV},
$$

which arises from fixing a single modulus to give the correct GUT scale coupling, $\alpha_{\mathrm{GUT}}=1 / 25$ arising from a 3 -cycle with volume $V_{X}=25$ in string units (see Appendix B 3). We model this by fixing the log-normal mean to $\log _{10} \bar{\mu}_{\mathrm{ax}}=-15$.
The fuzzy DM model [59-63] posits that DM composed of axions with mass

$$
\mu_{\mathrm{FDM}} \approx 10^{-22} \mathrm{eV}
$$

has certain desirable properties that could lead to its being favored over standard cold DM by observations of galactic structure. We model this by fixing the log-normal mean to $\log _{10} \bar{\mu}_{\mathrm{ax}}=-22$. The QCD axion [56-58] mass is given by

$$
\mu_{\mathrm{QCD}} \approx 6 \times 10^{-10} \mathrm{eV}\left(\frac{10^{16} \mathrm{GeV}}{f_{a}}\right) .
$$

In order to realize the QCD axion in M-theory, some light eigenstate in the "pure M-theory" spectrum should receive its mass dominantly from QCD instantons. Furthermore, the vacuum expectation value of this field should be not that far from $\theta=0$ to solve the strong- $C P$ problem. These two conditions together require that there is at least one eigenstate in the pure M-theory spectrum with [64]

$$
\mu_{\mathrm{ax}} \lesssim \mu_{\mathrm{ax}, \mathrm{low}} \approx 10^{-14} \mathrm{eV}
$$

We model this by fixing $\mu_{\mathrm{ax}}^{-}$and $\sigma$ such that $\mu_{\mathrm{ax}, \text { low }}$ is within $95 \%$ of the probability at the lower end of the distribution after $N_{\mathrm{ax}}$ draws. This fixes $\bar{\mu}_{\mathrm{ax}}\left(\sigma, N_{\mathrm{ax}}\right)$ in terms of standard error functions:

$$
N_{\mathrm{ax}} \operatorname{erfc}\left[-\frac{\log _{10}\left(\mu_{\mathrm{ax}, \mathrm{low}} / \bar{\mu}_{\mathrm{ax}}\right)}{\sqrt{2 \sigma^{2}}}\right]=0.1 .
$$

With the above fixed, one linear combination of axions receives its mass from QCD instantons. Therefore, we remove one axion from the $\mathrm{M}$-theory distribution and replace it with the QCD axion. The probability that the QCD axion in $\mathrm{M}$-theory is allowed based on BH superradiance data is thus

$$
P_{\mathrm{al}}\left(\sigma, N_{\mathrm{ax}}\right)=P_{\mathrm{al}}\left(\mu_{\mathrm{ax}, \mathrm{QCD}} \mid N_{\mathrm{ax}}=1\right)\left\{\int \mathrm{d} \mu_{\mathrm{ax}} p\left[\mu_{\mathrm{ax}} \mid \sigma, \bar{\mu}_{\mathrm{ax}}\left(\sigma, N_{\mathrm{ax}}\right)\right] P_{\mathrm{al}}\left(\mu_{\mathrm{ax}} \mid N_{\mathrm{ax}}=1\right)\right\}^{N_{\mathrm{ax}}-1} .
$$

Constraints on the distribution parameters of each of these benchmark models are shown in Fig. 15. While none of these models are ruled out for a single axion, in all cases the exclusion probability starts to become significant for nonzero distribution widths and large numbers of fields. In all cases, the maximum allowed value of $N_{\mathrm{ax}}$ increases for very large $\sigma$. For large $\sigma$ the distribution is approximately $\log$ flat with respect to the data exclusions, and increasing the width simply reduces the probability of overlap.

The GUT model has a small exclusion probability at zero width due to the large mass errors on the lightest SMBHs (NGC 4051 and MCG-6-30-15). The GUT model is excluded at better than the 95\% C.L. for all widths $\sigma<$ $\mathcal{O}(100)$ for $N_{\mathrm{ax}} \gtrsim 100$. The fuzzy DM model is excluded at better than the $95 \%$ C.L. for all widths $1 \lesssim \sigma \lesssim 10^{3}$ if $N_{\mathrm{ax}} \gtrsim 100$.

The QCD axion model is the least constrained by the data. The mass of the QCD axion with $f_{a} \gtrsim 10^{17} \mathrm{GeV}$ is not excluded itself by BH superradiance, nor is the light mass $\mu_{\mathrm{ax}, \text { low }}$ required from the M-theory part of the spectrum. There is a small range of intermediate widths where the distribution does overlap the excluded region, excluding $0.2 \lesssim \sigma \lesssim 4$ if $N_{\mathrm{ax}} \gtrsim 100$ at $95 \%$ C.L. while $\sigma \gtrsim 4$ is allowed for all $N_{\mathrm{ax}}<1000$ considered. 


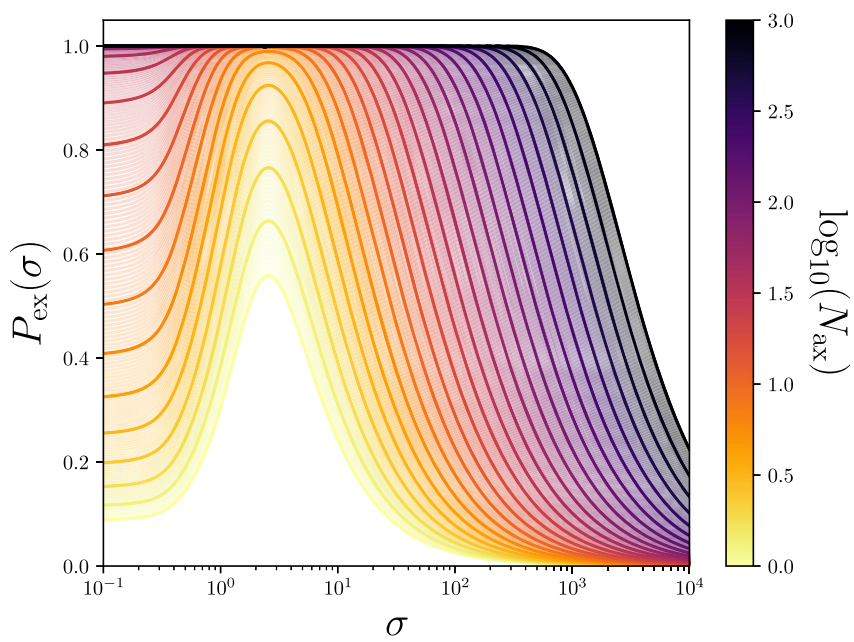

(a) GUT.

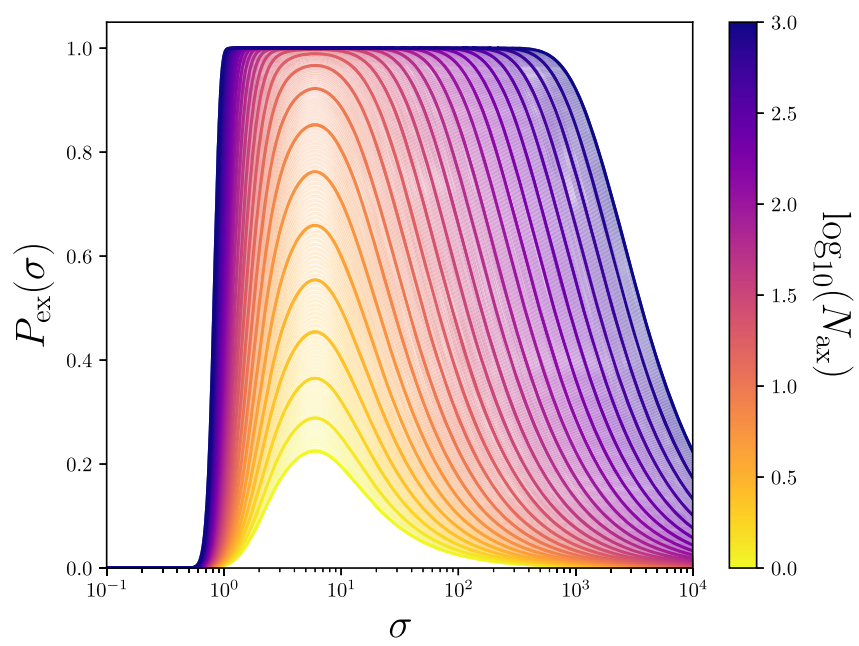

(b) Fuzzy DM.

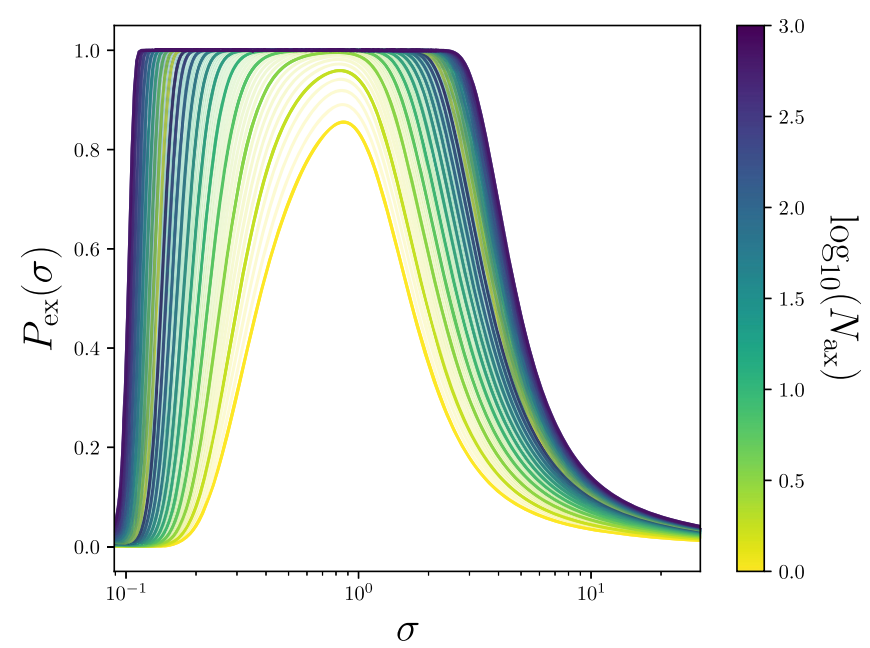

(c) $\mathrm{QCD}$.

FIG. 15. Probability of exclusion as a function of the dimensionless spread, $\sigma$ determined by the model variance defining the mass spectra in the M-theory axiverse for $N_{\mathrm{ax}}=1 \rightarrow 1000$. Each panel corresponds to three unique scenarios which determine the mean of the mass spectrum required to maximize the probability of drawing the desired masses detailed in Sec. IV C 2 . In the limit $\sigma \gg 1$ the total probability for $N_{\mathrm{ax}}=1 \rightarrow \infty$ converges to zero as the spread crosses the bounds deemed probable by BH spin measurements. The behavior in the limit $\sigma \ll 1$ is determined by the accuracy of the available BH mass/spin measurements.

\section{Comment on fuzzy $\mathrm{DM}$ and $\mathrm{BH}$ superradiance}

Recently it has been claimed that the global $21-\mathrm{cm}$ signal [109], which is strong evidence that the Universe was undergoing reionization at redshift $z_{\text {re }} \approx 17$, places a lower bound on the fuzzy DM mass of $\mu_{\mathrm{ax}} \geq 5-8 \times 10^{-21} \mathrm{eV}$ $[110,111]$. This result is extremely interesting since, if its accuracy is to be believed, it significantly shrinks the gap between fuzzy DM bounds from $\mathrm{BH}$ superradiance and structure formation. In the context of the present work, if fuzzy DM is realized from a mass distribution, then respecting the reionization bound and $\mathrm{BH}$ superradiance demands an extremely narrow distribution with a small number of light fields. If the gap between fuzzy DM constraints from $\mathrm{BH}$ superradiance and reionization is closed, either by the measurement of spins of the most massive SMBHs, or improvements on the lower limit to $z_{\text {re }}$, then fuzzy DM with no self-interactions will be completely excluded. Rescuing fuzzy DM from BH superradiance constraints in such a case would require selfinteraction strengths corresponding to decay constants $f_{a} \lesssim 10^{16} \mathrm{GeV}$. Low decay constants open the door to new fuzzy DM phenomenology [112-114], but may become increasingly hard to realize in small-volume string compactifications.

\section{DISCUSSION AND CONCLUSIONS}

BH superradiance places strong constraints on the possible existence of light bosonic fields with small selfinteractions, in particular on axionlike fields. Many authors 
have considered these constraints for the case of a single new light field. The excluded ranges of axion mass are

$$
\begin{aligned}
& 7 \times 10^{-14} \mathrm{eV}<\mu_{\mathrm{ax}}<2 \times 10^{-11} \mathrm{eV}, \\
& 7 \times 10^{-20} \mathrm{eV}<\mu_{\mathrm{ax}}<1 \times 10^{-16} \mathrm{eV} .
\end{aligned}
$$

A model with multiple axions is excluded if just one field lies in these ranges. We have studied this possibility, and used $\mathrm{BH}$ superradiance to exclude certain distributions of axion masses. The constraints become more severe with larger numbers of axionlike fields due to the increased probability of drawing an outlier. This allows us to place constraints on the number of axionlike fields, $N_{\mathrm{ax}}$.

Models for axions coming from string theory and M-theory typically involve many axionlike fields. These fields have their masses determined by microscopic quantities related to the geometry of the compact space. Their masses, however, are expected to follow particular statistical distributions independently of the microscopic details. We have considered various different distributions-log-flat, log-normal, and Marčhenko-Pastur-using BH superradiance to bound both the parameters of the distribution, and, more significantly, the number of light axions within that distribution.

Constraints on $N_{\text {ax }}$ from a process such as $\mathrm{BH}$ superradiance, which relies only on the existence of the vacuum fluctuations of the given field, are extremely powerful, and could be used in this context to bound the dimensionality of phenomenologically consistent moduli spaces in string/ M-theory. Indeed we have seen that the benchmark value of $N_{\mathrm{ax}} \approx 30$ found in the majority of known Calabi-Yau manifolds can be excluded for a wide range of distribution parameters. Only a small number of fields should obtain masses anywhere in the $\mathrm{BH}$ superradiance region from $10^{-10} \mathrm{eV} \lesssim \mu_{\mathrm{ax}} \lesssim 10^{-20} \mathrm{eV}$, which can be accommodated with a single very wide distribution $\sigma \gtrsim 30$, or bimodal distributions containing only very light or relatively heavy axions.

Our analysis has neglected axion self-interactions, which shut off BH superradiance if they are strong, and other constraints, e.g., coming from the relic abundance. It would be interesting in this regard to combine our previous analysis in Ref. [65] with the current analysis and compute, in addition to axion masses, the axion decay constants, relic density, and self-interaction potential. The present work is more model independent, since it does not rely on any cosmological assumptions, and applies to any model for light scalars with sufficiently small self-interactions. The extended and combined analysis will be the subject of future work.

\section{ACKNOWLEDGMENTS}

We acknowledge useful conversations with Bobby Acharya, Katy Clough and Chakrit Pongkitivanichkul.
The work of M.J.S. is supported by funding from the UK Science and Technology Facilities Council (STFC). D. J. E. M. is supported by the Alexander von Humboldt Foundation and the German Federal Ministry of Education and Research.

\section{APPENDIX A: STATISTICAL MODEL}

We model the BH data in Table I with two-dimensional multivariate Gaussian distributions for both $x=M_{\mathrm{BH}}$ and $y=a_{*}$. There are $N_{d}$ data points $d_{i}$ comprising the data set $\left\{d_{i}\right\}$. For each point in the data set the values of $M_{\mathrm{BH}}$ and $a_{*}$ and their associated errors become centered data values $(\bar{x}, \bar{y})$ with errors $\left(\sigma_{x}, \sigma_{y}\right)$. We are interested in the probability that a given model, $\mathcal{M}$, is excluded given the data, $\left\{d_{i}\right\}$ : $P_{\text {ex }}\left(\mathcal{M} \mid\left\{d_{i}\right\}\right)$. Since a single data point in the disallowed region would exclude the model, $P_{\text {ex }}\left(\mathcal{M} \mid\left\{d_{i}\right\}\right)$ is given by the probability that any single data point is above the $\mathrm{BH}$ superradiance isocontour boundaries for each value of $l$. For a large number of data points, this is a relatively tricky combinatorial problem. However, the probability is normalized such that

$$
P_{\text {ex }}\left(\mathcal{M} \mid\left\{d_{i}\right\}\right)=1-P_{\text {allowed }}\left(\mathcal{M} \mid\left\{d_{i}\right\}\right) .
$$

Now we can use the binomial theorem (or a simple probability tree) to note that $P_{\text {allowed }}\left(\mathcal{M} \mid\left\{d_{i}\right\}\right)$ is simply the cumulative probability that all data points simultaneously fluctuate below the isocontour,

$$
P_{\text {allowed }}\left(\mathcal{M} \mid\left\{d_{i}\right\}\right)=\prod_{i} P_{\text {allowed }}\left(\mathcal{M} \mid d_{i}\right),
$$

and $P_{\text {allowed }}\left(\mathcal{M} \mid d_{i}\right)$ is simply the volume of the bivariate Gaussian contained outside the isocontour boundary given by the function $y=f(x)$.

To evaluate $P_{\text {allowed }}\left(\mathcal{M} \mid d_{i}\right)$ in a numerically efficient manner, we make two simplifying assumptions. First, we assume zero covariance between $x$ and $y$. Second, the error on the two-dimensional data can be evaluated using an effective one-dimensional error $[115,116]$. These two simplifications allow us to use the standard error function to evaluate $P_{\text {allowed }}\left(\mathcal{M} \mid d_{i}\right)$, rather than the more numerically expensive integral under the curve. The shape of the BH superradiance contours $y=f(x)$, which only have support over finite $x$, requires this procedure to be evaluated in two separate regimes. Where the contour is defined, we use the contour as $y=f(x)$ and evaluate the effective one-dimensional error in $y, \Sigma_{y}$, as

$$
\Sigma_{y}^{2}=\sigma_{y}^{2}+f^{\prime}(\bar{x})^{2} \sigma_{x}^{2} .
$$

When the contour is not defined for a given $x$, we instead use the inverse function $x=g(y)$ and evaluate the effective error in $x, \Sigma_{x}$, as 


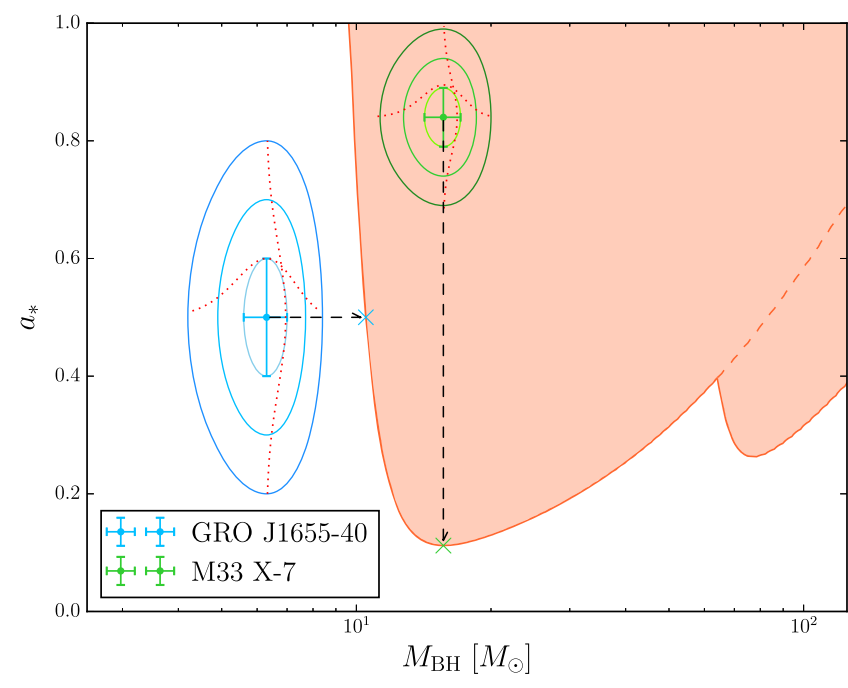

FIG. 16. Visual representation of the statistical model methodology using two example stellar BHs, GRO J1655-40 and M33 $\mathrm{X}-7$ with instability bounds for an axion mass, $\mu_{\mathrm{ax}}=10^{-12.75} \mathrm{eV}$. Each data point is shown with $1 \sigma, 2 \sigma$ and $3 \sigma$ contour levels. Effective errors are calculated by projection on either the $x$ or $y$ axis (crossed points) depending on weather the $\mathrm{BH}$ falls inside the instability bounds where $f(x)$ is defined.

$$
\Sigma_{x}^{2}=\sigma_{x}^{2}+g^{\prime}(\bar{y})^{2} \sigma_{y}^{2}
$$

The effective errors are represented visually in Fig. 16. Since our functions are all given numerically, the inverse function and its derivative are trivial to evaluate given the original function.

A complication arises since $g(y)$ is multivalued, taking two values $g_{1}$ and $g_{2}$ for a single $y$. We choose to evaluate the derivative $g^{\prime}(\bar{y})$ at the nearest part of the contour [i.e., the value $g_{i}$ which minimizes $\left.\bar{x}-g(\bar{y})\right]$, and evaluate the error function between the two values $g_{1}$ and $g_{2}$. This approximation only affects $P_{\text {allowed }}\left(\mathcal{M} \mid d_{i}\right)$ for values close to unity, while $P_{\text {ex }}\left(\mathcal{M} \mid\left\{d_{i}\right\}\right)$ is dominated by the smallest values of $P_{\text {allowed }}\left(\mathcal{M} \mid d_{i}\right)$ contained well within the contours where $f(x)$ has support and is single valued.

The use of the effective errors (A3) and (A4) assumes that, for a given data point, the functions $f(x)$ and $g(y)$ are smooth at the mean value over the range of the errors. When a $\mathrm{BH}$ data point with large errors sits close to a cusp in the contours the exclusion probability computed from the effective error is smaller than the true answer. Cusps in the total contour are caused by the meeting of individual contours with different $l$ values, each of which are smooth. A more exact procedure would thus be to compute the probability individually for each $l$ contour, and then compute the cumulative probability from a product over $l$. This would increase the number of likelihood evaluations by $l_{\max } \times N_{d}$, and for speed of computation we do not perform this more accurate calculation. The more accurate calculation would give larger exclusion probabilities (reducing the overall effective size of $\mathrm{BH}$ errors), and so the approximate computation is more conservative in the sense that it does not give overly strong exclusions.

\section{APPENDIX B: THE AXIVERSE MASS SPECTRUM}

\section{Diagonalizing the Lagrangian}

The most general form for the multiaxion action for fields below any compactification, moduli stabilization or Peccei-Quinn (PQ) symmetry scales is of the form given in Eq. (23). We set the axion field alignment used to determine the diameter of the fundamental domain to only include $P=N$, where we always possess sufficient instanton contributions $N$ for each axion field, $P$. We restrict our considerations to nonperturbative terms with trivial charges, $\mathcal{Q}_{j, i}=\mathbb{1}_{N}$. We therefore only need to consider enhancements to the $N_{\mathrm{ax}}$ field space diameter defined as the longest distance between vertices in the polytopes defining the field ranges via the Pythagorean sum from the $\mathrm{N}$-flation model and kinetic alignment in our models considered in Appendix B 2. Lattice alignment as well as alignment theories possessing $P \geq N$ are beyond the scope of this work. See Refs. [85,117-119] for extensive details of axion field alignment.

We begin in the lattice basis, with an axion defined by a single cosine potential possessing a shift symmetry obeying, $\theta_{i} \rightarrow \theta_{i}+2 \pi$. We diagonalize and canonically normalize the axion field space metric $\mathcal{K}_{i j}$ moving to the kinetic basis with the unitary rotation $U_{i j}$ where,

$\mathcal{K}_{i j}=U_{i k}^{T} \operatorname{diag}\left(\mathcal{K}_{k l}\right) U_{l j}=\frac{1}{2} U^{T} \operatorname{diag}\left(f_{a}\right) \operatorname{diag}\left(f_{a}\right) U$.

We define the axion decay constants, $f_{a}$, from the eigenvalues of $\mathcal{K}_{i j}$ in the lattice basis in Planck units,

$$
\vec{f}_{a}=\sqrt{2 \operatorname{eig}\left(\mathcal{K}_{i j}\right)} .
$$

We can now define the canonically normalized field as,

$$
\tilde{\phi}_{i}=M_{p l} \operatorname{diag}\left(f_{a}\right) U_{i j} \theta_{j} .
$$

In the kinetic basis the effective Lagrangian takes the form,

$$
\mathcal{L}=-\frac{1}{2} \partial_{\mu} \tilde{\phi}_{i} \partial^{\mu} \tilde{\phi}_{j}-\frac{1}{2} \tilde{\phi}_{i} \tilde{\mathcal{M}}_{i j} \tilde{\phi}_{j}
$$

where the new mass matrix is defined as,

$$
\tilde{\mathcal{M}}=2 \operatorname{diag}\left(1 / f_{a}\right) U \mathcal{M} U^{T} \operatorname{diag}\left(1 / f_{a}\right) .
$$

Moving to the mass eigenstate basis with a further unitary rotation, $V_{i j}$ gives,

$$
\tilde{\mathcal{M}}=V^{T} \operatorname{diag}\left(m_{a}^{2}\right) V .
$$


In this basis the mass eigenstate fields are defined as,

$$
\phi=V \tilde{\phi}=M_{p l} V \operatorname{diag}\left(f_{a}\right) U \theta,
$$

with the effective Lagrangian,

$$
\mathcal{L}=-\frac{1}{2} \partial_{\mu} \phi_{i} \partial^{\mu} \phi_{j}-\frac{1}{2} \phi_{i} \mathcal{M}_{i j} \phi_{j}
$$

\section{The random matrix theory mass spectrum}

A systematic construction of the axion decay constant and mass spectrum in explicit realizations of the string axiverse is an extremely complex and numerically comprehensive task to undertake. In general, considerations need to be made for leading instanton corrections to the superpotential [Eq. (B10)], a calculation of the full scalar potential along with a minimization of polynomial expressions with potentially many variables when considering realistic numbers of apparent axions or moduli. See Ref. [120] for a detailed discussion of the complexities of the string landscape. The effective field theory approach in Appendix B 1 can benefit from the simplistic nature of RMT-inspired models on the grounds of universality. In these models universality dictates that the distribution of the physical dimensional parameters are characterized by some mean scale and variance. For generic field space metric considerations the kinetic matrix in Eq. (23) can be well described by a matrix belonging to a class of matrices of the Wishart form,

$$
X_{i j}=\frac{1}{N} Y_{i k}^{T} Y_{k j}
$$

This formalism can also be extended in the small-field approximation to govern the properties of the axion mass matrix. The mean scale of the axion population defines the phenomenological properties of the fields. The spread of the spectrum is controlled by a shaping index $\beta_{\mathcal{K}, \mathcal{M}} \in(0,1]$ which has been shown to have theoretical foundations related to the total dimension of the moduli space $[65,81]$. Below we present a series of RMT-inspired models based on charge quantization and field space alignment considerations.

The defining features of each of our models we consider are presented in Table II. Each model presents a modest hierarchy in complexity regarding the initial basis and determined mass eigenstate spectrum. Our first model is based on $N$-flation type field alignment [81] acting as our simplest strawman model. The fields in canonical coordinates are defined by the effective field space metric, $\mathcal{K}_{i j}=\operatorname{diag}\left(f_{a}^{2}\right)$. The canonical field ranges in this basis are defined as $\phi_{i}=\bar{f}_{a} \theta_{i}$ where $\bar{f}_{a}$ is a scaling factor introduced to represent the degenerate decay constant scales arising from the diagonal kinetic matrix. The field space diameter is given by the pythagorean sum over the $\mathrm{N}$-dimensional hyper-rectangle (see Table II). The resulting mass spectra are displayed in Fig. 7.

When beginning in the lattice basis a spectrum of decay constants now scale the initial field sampling. It has been shown that the kinetic matrix $\mathcal{K}_{i j}$, could belong to the Gaussian orthogonal Wishart ensemble with independent and identically distributed Gaussian entries [85,121]. The entries for the submatrices $Y_{i j}$ in Eq. (B9) composing each of the kinetic and mass matrices are drawn from normal distributions with zero mean and unit variance. The axion decay constant spectrum is given by the limiting MarčhenkoPastur law (Fig. 7). In the mass eigenstate basis the mass spectrum is now rotated by the nontrivial rotations between the lattice and kinetic basis. Universality dictates a convergent mass spectrum well modeled by a log-normal distribution with its limited variance, $\sigma$ defined by the bounded nature of the initial Wishart structure. The mass spectrum in this model is presented in Fig. 17(a).

If the entries of the submatrices are not selected as independent and identically distributed Gaussian entries and instead selected from a log-uniform distribution, the resulting matrix of the Wishart form will now reside in a class of rank-one spiked Wishart matrices. In the original models [122-124] the matrices are defined by the class, $W_{R}(\Sigma, M)$ where a single element of the covariance matrix deviates from unity inducing a phase transition in the distribution for the largest eigenvalues. In the limit $N_{\mathrm{ax}} \rightarrow \infty$ a bulk region forms supported by the Marčhenko-Pastur limiting law and one singular eigenvalue is repulsed from the bulk as shown in Fig. 17(b). The approximate order of the singular eigenvalue is $\lambda_{\mathrm{ax}} \approx \mathcal{O}\left(N_{\mathrm{ax}}\right)$, which defines the enhancement of the diameter of field space by a factor of $\sqrt{N_{\mathrm{ax}}}$ due to the large hierarchy between $f_{\mathrm{a} \text {,max }}$ and the second largest eigenvalue. This behavior governs the axion decay constant spectrum in the model (up to canonical normalization factors) as shown in Fig. 17(c). In the limit

TABLE II. RMT models considered in this work and extensively covered in Ref. [65]. Detailed are the initial basis considerations for each effective model along with the relevant sampling procedures and field space diameters. The values of $\sigma$ represent the approximate spread a population or subpopulation of axions would have in each model.

\begin{tabular}{lcccccc}
\hline \hline Model & Alignment & Initial Basis & Sampling & Diameter & Spectra & $\sigma$ \\
\hline Marčhenko-Pastur & N-flation & Kinetic Eq. (B8) & $B_{i j} \in \mathcal{N}(0,1)$ & $D=2 \pi \sqrt{N_{\text {ax }} \bar{f}_{a}^{2}}$. & Fig. 7 & $\sim \mathcal{O}(1)$ \\
White Wishart & Kinetic & Lattice Eq. (23) & $A_{i j}, B_{i j} \in \mathcal{N}(0,1)$ & $D=2 \pi \sqrt{N_{\text {ax }} f_{\text {a,max }} .}$ & Fig. 17(a) & $\sim(0.1-5)$ \\
Spiked Wishart & Kinetic & Lattice Eq. (23) & $A_{i j}, B_{i j} \in \log _{10} \mathcal{U}($ min, max $)$ & $D=2 \pi \sqrt{N_{\text {ax }} f_{\text {a,max }} .}$ & Fig. 17(c) & $\sim(0.1-5)$ \\
\hline \hline
\end{tabular}




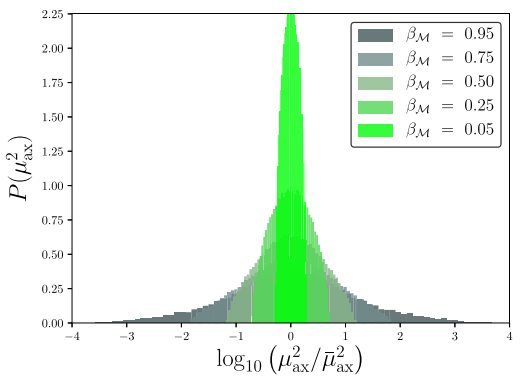

(a) White Wishart class mass eigenstate basis spectrum.

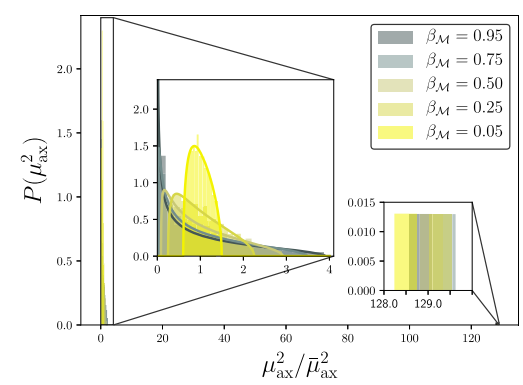

(b) Spiked Wishart class kinetic basis spectrum.

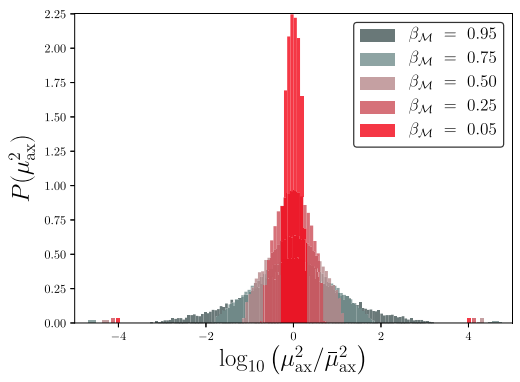

(c) Spiked Wishart class mass eigenstate basis spectrum.

FIG. 17. Normalized eigenvalue spectra for axion masses $\mu_{\mathrm{ax}}^{2}$ for different values of the spectrum-shaping parameter $\beta_{\mathcal{M}}$ in each Wishart class model in Table II.

$\beta_{\mathcal{M}}=1$ the mass spectra converges to the white Wishart case. For values of $\beta_{\mathcal{M}}<1$ the convergent mass spectrum is well modeled by a log-normal distribution plus two positively and negatively logarithmically repulsed regions enhancing the total spectral width.

\section{The M-theory mass spectrum}

It has been shown in M-theory compactified on $G_{2}$ manifolds with an absence of fluxes that it is possible to stabilize both the moduli and axions in order to realize a spectrum of ultralight axions in the low-energy spectrum of its four-dimensional effective supergravity theory. We follow the explicit realization of the string axiverse in Refs. $[64,125,126]$. In such models the moduli are stabilized in a nonsupersymmetric minimum, with all axions pairing up with geometric moduli where all moduli superfields possess PQ symmetries. The superpotential in this model takes the form

$$
W=A_{1} \phi_{1}^{a} e^{i b_{1} F_{1}}+A_{2} e^{i b_{2} F 1}+\sum_{k=3}^{\infty} A_{k} e^{i b_{k} F_{k}}
$$

with order $\mathcal{O}(1)$ constants, $A_{k}$. The first two terms in Eq. (B10) come from strong gauge dynamics in the hidden sector using up one combination of axions where $\phi_{1}$ is a holomorphic composite field made of hidden sector matter fields. In general the rest of the fields present in the summation come from nonperturbative physics such as membrane instantons and serve as a fundamental feature of such compactification models. We only need to consider the higher-order correctional terms assumed to be generated from membrane instantons where $b_{k}=2 \pi I$ and $I \in \mathbb{Z}$ with the gauge kinetic functions, $F_{k}=\sum_{i=1}^{N} N_{K}^{i} z_{i}$. It is always possible to find realistic arguments determining the number of nonperturbative effects as larger than the number of axions, $N_{\text {Inst }}>N_{\text {ax }}$ giving rise to sufficient independent terms in the superpotential. To consider a spectrum of axions we integrate out the moduli and heavy axion combinations such that the relevant effective superpotential becomes

$$
W_{\text {Inst }}=\sum_{i=1}^{N} \tilde{\Lambda}_{i}^{3} e^{i b_{i} F_{i}},
$$

where $\tilde{\Lambda}_{i}$ are the associated mass scales for each nonperturbative effect. The potential now takes the following form:

$$
\begin{aligned}
V & \approx F\left(\sum_{i=1}^{N_{\mathrm{ax}}} \frac{\partial}{\partial z_{i}} \sum_{j=1}^{N} \tilde{\Lambda}_{j}^{3} e^{i b_{j} F_{j}}\right)+\text { c.c. } \\
& \approx \sum_{i=1}^{N_{\mathrm{ax}}} \sum_{j=1}^{N} \frac{2 F \tilde{\Lambda}_{j}^{3} b_{j} N_{j}^{i}}{M_{S}} e^{-b_{j} \sum_{k}^{N_{\mathrm{ax}}} N_{j}^{k} s_{k}} \cos \left(\sum_{k=1}^{N_{\mathrm{ax}}} b_{j} N_{j}^{k} t_{k}\right) .
\end{aligned}
$$

In Ref. [65] it was shown that an expansion of the periodic potentials to quadratic order reveals the axion mass matrix,

$\mathcal{M}_{i j}=\sum_{k=1}^{N_{\mathrm{ax}}} \sum_{r=1}^{N} \frac{4 F \tilde{\Lambda}_{r}^{3} b_{r} N_{r}^{k}}{M_{S}^{3}} e^{-b_{r} \sum_{m}^{N_{\mathrm{ax}}} N_{r}^{m} s_{m}} b_{r} N_{r}^{i} b_{r} N_{r}^{j}$,

$$
=\sum_{r=1}^{N} \frac{4 F \tilde{\Lambda}_{r}^{3} C_{r}}{M_{S}^{3}} e^{-S_{r}} \tilde{N}_{r}^{i} \tilde{N}_{r}^{j},
$$

where $\tilde{N}_{i}^{j}=b_{i} N_{i}^{j}$ is a rectangular matrix of size $\left(N_{\mathrm{ax}}, N\right)$, $C_{r}=\sum_{k}^{N_{\mathrm{ax}}} \tilde{N}_{r}^{k}$ and $S_{r}=\sum_{m}^{N_{\mathrm{ax}}} \tilde{N}_{r}^{m} s_{m}$. The dimensions of $\tilde{N}_{i}^{j}$ are controlled by the axion population size, $N_{\text {ax }}$ and the number of nonperturbative instantons, $N$. Using this form the mass matrix can be parametrized as

$$
\mathcal{M}_{i j}=\sum_{r=1}^{N} 4 F \tilde{\Lambda}_{r}^{3} C_{r} e^{-S_{r}} \tilde{N}_{r}^{i} \tilde{N}_{r}^{j},
$$

which in terms of submatrix structure following the philosophy of Eq. (B9) gives, 


$$
\mathcal{M}_{i j}=\frac{1}{N} A_{i r} A_{j r}
$$

This defines the following form for the sample submatrix:

$$
A_{i r}=\left(2 \sqrt{F \tilde{\Lambda}_{r}^{3} C_{r}}\right) e^{-S_{r} / 2} \tilde{N}_{r}^{i}
$$

where $i, j=1, \ldots, N_{\mathrm{ax}}, r=1, \ldots, N$, and $A_{i r}$ is a rectangular matrix of size $\left(N_{\mathrm{ax}}, N\right)$ with a normalization factor $1 / N$.

In order to define our mass scales of interest in the M-theory axiverse we consider the general form for the superpotential in Eq. (B11). $F_{i}$ represents the gauge kinetic functions which are linear combinations of the moduli superfields,

$$
F_{i}=\sum_{k}^{n_{\mathrm{ax}}} N_{i}^{k} z_{k}=\sum_{k}^{n_{\mathrm{ax}}} N_{i}^{k}\left(t_{k}+i s_{k}\right) .
$$

The generalized volume of the corresponding 3-cycles is calculated from,

$$
V_{X}^{i}=\operatorname{Im}\left(F_{i}\right)=\sum_{k=1}^{n_{\mathrm{ax}}} N_{i}^{k} s_{k}=\frac{1}{2 \pi} \sum_{k=1}^{n_{\mathrm{ax}}} \tilde{N}_{i}^{k} s_{k} .
$$

The geometric moduli are stabilized in terms of a single parameter $\left\langle V_{X}\right\rangle$ which represents the stabilized volume of the 3-cycle supporting the hidden sector. In order to realize a GUT in the low-energy limit of the theory, at least one of the gauge kinetic functions must give rise to the expected value of the GUT coupling constant,

$$
\alpha_{\mathrm{GUT}}=\frac{1}{V_{X}} \approx \frac{1}{25} .
$$

The average value of $\left\langle V_{X}\right\rangle$ therefore fixes the mass scales of the spectrum of axions appearing in the visible sector (Fig. 8). We parametrize the axion mass distribution in terms of the average value of the 3-cycle volume distribution, $\left\langle V_{X}\right\rangle$ via the relationship

$$
\left\langle V_{X}\right\rangle=\frac{N_{\mathrm{ax}} \tilde{N}_{\max }\langle s\rangle}{4 \pi},
$$

which contains the parameters we statistically sample to determine the nature of $\sigma$ used throughout the basis of this work (see Sec. III D of Ref. [65] for details of the parameters used). The number of parameters and hierarchy of scales involved in the statistical sampling of the 3-cycle volume dictate a large spread in the mass eigenstates covering many decades. The nature of universality ensures a convergence to a normal distribution over these scales [65].
[1] R. Penrose, Gravitational collapse: The role of general relativity, Riv. Nuovo Cimento Soc. Ital. Fis. 1, 252 (1969).

[2] W. H. Press and S. A. Teukolsky, Floating orbits, superradiant scattering and the black-hole bomb, Nature (London) 238, 211 (1972).

[3] W. H. Press and S. A. Teukolsky, Perturbations of a rotating black hole. II. Dynamical stability of the Kerr Metric, Astrophys. J. 185, 649 (1973).

[4] V. Cardoso, O. J. C. Dias, J. P. S. Lemos, and S. Yoshida, The black hole bomb and superradiant instabilities, Phys. Rev. D 70, 044039 (2004); Erratum, Phys. Rev. D70, 049903(E) (2004).

[5] R. Brito, V. Cardoso, and P. Pani, Superradiance (Springer, New York, 2015).

[6] L. Barack et al., Black holes, gravitational waves and fundamental physics: A roadmap, arXiv:1806.05195.

[7] A. Arvanitaki, M. Baryakhtar, S. Dimopoulos, S. Dubovsky, and R. Lasenby, Black hole mergers and the QCD axion at Advanced LIGO, Phys. Rev. D 95, 043001 (2017).

[8] M. Baryakhtar, R. Lasenby, and M. Teo, Black hole superradiance signatures of ultralight vectors, Phys. Rev. D 96, 035019 (2017).
[9] R. Brito, S. Ghosh, E. Barausse, E. Berti, V. Cardoso, I. Dvorkin, A. Klein, and P. Pani, Gravitational wave searches for ultralight bosons with LIGO and LISA, Phys. Rev. D 96, 064050 (2017).

[10] V. Cardoso, Ó. J. C. Dias, G. S. Hartnett, M. Middleton, P. Pani, and J.E. Santos, Constraining the mass of dark photons and axion-like particles through black-hole superradiance, J. Cosmol. Astropart. Phys. 03 (2018) 043.

[11] O. A. Hannuksela, R. Brito, E. Berti, and T. G.F. Li, Probing the existence of ultralight bosons with a single gravitational-wave measurement, arXiv:1804.09659.

[12] A. Arvanitaki and S. Dubovsky, Exploring the string axiverse with precision black hole physics, Phys. Rev. D 83, 044026 (2011).

[13] M. Baryakhtar, R. Lasenby, and M. Teo, Black hole superradiance signatures of ultralight vectors, Phys. Rev. D 96, 035019 (2017).

[14] R. Brito, S. Ghosh, E. Barausse, E. Berti, V. Cardoso, I. Dvorkin, A. Klein, and P. Pani, Gravitational wave searches for ultralight bosons with LIGO and LISA, Phys. Rev. D 96, 064050 (2017).

[15] D. Baumann, H. S. Chia, and R. A. Porto, Probing ultralight bosons with binary black holes, arXiv:1804.03208. 
[16] R. Brito, V. Cardoso, and P. Pani, Black holes as particle detectors: evolution of superradiant instabilities, Classical Quantum Gravity 32, 134001 (2015).

[17] H. Yoshino and H. Kodama, Gravitational radiation from an axion cloud around a black hole: Superradiant phase, Prog. Theor. Exp. Phys. 2014, 043 E02 (2014).

[18] P. Pani, V. Cardoso, L. Gualtieri, E. Berti, and A. Ishibashi, Black-Hole Bombs and Photon-Mass Bounds, Phys. Rev. Lett. 109, 131102 (2012).

[19] H. Kodama, Superradiance and instability of black holes, Prog. Theor. Phys. Suppl. 172, 11 (2008).

[20] A. Arvanitaki, S. Dimopoulos, S. Dubovsky, N. Kaloper, and J. March-Russell, String axiverse, Phys. Rev. D 81, 123530 (2010).

[21] A. Arvanitaki, M. Baryakhtar, and X. Huang, Discovering the QCD Axion with Black Holes and Gravitational Waves, Phys. Rev. D 91, 084011 (2015).

[22] M. Middleton, Black Hole Spin: Theory and Observation (Springer, New York, 2016), pp. 99-151.

[23] M. Coleman Miller and J. M. Miller, The masses and spins of neutron stars and stellar-mass black holes, Phys. Rep. 548, 1 (2015).

[24] L. W. Brenneman, C. S. Reynolds, M. A. Nowak, R. C. Reis, M. Trippe, A. C. Fabian, K. Iwasawa, J. C. Lee, J. M. Miller, R. F. Mushotzky, K. Nandra, and M. Volonteri, The spin of the supermassive black hole in NGC 3783, Astrophys. J. 736, 103 (2011).

[25] C. S. Reynolds, Measuring black hole spin using x-ray reflection spectroscopy, Space Sci. Rev. 183, 277 (2014).

[26] C. S. Reynolds, The spin of supermassive black holes, Classical Quantum Gravity 30, 244004 (2013).

[27] B. P. Abbott et al. (Virgo and LIGO Scientific Collaborations), Binary Black Hole Mergers in the First Advanced LIGO Observing Run, Phys. Rev. X 6, 041015 (2016).

[28] B. P. Abbott et al. (Virgo and LIGO Scientific Collaborations), GW170104: Observation of a 50-Solar-Mass Binary Black Hole Coalescence at Redshift 0.2, Phys. Rev. Lett. 118, 221101 (2017).

[29] J. A. Orosz, J. E. McClintock, J. P. Aufdenberg, R. A. Remillard, M. J. Reid, R. Narayan, and L. Gou, The mass of the black hole in Cygnus X-1, Astrophys. J. 742, 84 (2011).

[30] L. Gou, J. E. McClintock, R. A. Remillard, J. F. Steiner, M. J. Reid, J. A. Orosz, R. Narayan, M. Hanke, and J. García, Confirmation via the continuum-fitting method that the spin of the black hole in Cygnus X-1 is extreme, Astrophys. J. 790, 29 (2014).

[31] J. A. Orosz, J. F. Steiner, J. E. McClintock, M. A. P. Torres, R. A. Remillard, C. D. Bailyn, and J. M. Miller, An improved dynamical model for the microquasar XTE J1550-564, Astrophys. J. 730, 75 (2011).

[32] J. F. Steiner, R. C. Reis, J. E. McClintock, R. Narayan, R. A. Remillard, J. A. Orosz, L. Gou, A. C. Fabian, and M. A.P. Torres, The spin of the black hole microquasar XTE J1550-564 via the continuum-fitting and Fe-line methods, Mon. Not. R. Astron. Soc. 416, 941 (2011).

[33] A. G. Cantrell, C. D. Bailyn, J. A. Orosz, J. E. McClintock, R. A. Remillard, C. S. Froning, J. Neilsen, D. M. Gelino, and L. Gou, The inclination of the soft x-ray transient
A0620-00 and the mass of its black hole, Astrophys. J. 710, 1127 (2010).

[34] L. Gou, J. E. McClintock, J. F. Steiner, R. Narayan, A. G. Cantrell, C. D. Bailyn, and J. A. Orosz, The spin of the black hole in the soft x-ray transient A0620-00, Astrophys. J. Lett. 718, L122 (2010).

[35] A Massive Star Odyssey: From Main Sequence to Supernova, edited by A. Herrero, J. A. Orosz, K. van der Hucht, and C. Esteban (Astronomical Society of the Pacific, San Francisco, 2003), p. 365.

[36] R. Shafee, J. E. McClintock, R. Narayan, S. W. Davis, L.-X. Li, and R. A. Remillard, Estimating the spin of stellar-mass black holes via spectral fitting of the X-ray continuum, Astrophys. J. 636, L113 (2006).

[37] R. Shafee, J. E. McClintock, R. Narayan, S. W. Davis, L.-X. Li, and R.A. Remillard, Estimating the spin of stellar-mass black holes by spectral fitting of the X-ray continuum, Astrophys. J. Lett. 636, L113 (2006).

[38] J. Greene, C. D. Bailyn, and J. A. Orosz, Optical and infrared photometry of the micro-quasar GRO J1655-40 in quiescence, Astrophys. J. 554, 1290 (2001).

[39] D. Steeghs, J. E. McClintock, S. G. Parsons, M. J. Reid, S. Littlefair, and V. S. Dhillon, The not-so-massive black hole in the microquasar GRS1915 + 105, Astrophys. J. 768, 185 (2013).

[40] J. E. McClintock, R. Shafee, R. Narayan, R. A. Remillard, S. W. Davis, and L.-X. Li, The spin of the near-extreme Kerr black hole GRS1915 + 105, Astrophys. J. 652, 518 (2006).

[41] J. A. Orosz et al., A new dynamical model for the black hole binary LMC X-1, Astrophys. J. 697, 573 (2009).

[42] L. Gou, J. E. McClintock, J. Liu, R. Narayan, J. F. Steiner, R. A. Remillard, J. A. Orosz, S. W. Davis, K. Ebisawa, and E. M. Schlegel, A determination of the spin of the black hole primary in LMC X-1, Astrophys. J. 701, 1076 (2009).

[43] J. A. Orosz, J. F. Steiner, J. E. McClintock, M. M. Buxton, C. D. Bailyn, D. Steeghs, A. Guberman, and M. A. P. Torres, The mass of the black hole in LMC X-3, Astrophys. J. 794, 154 (2014).

[44] J. F. Steiner, J. E. McClintock, J. A. Orosz, R. A. Remillard, C. D. Bailyn, M. Kolehmainen, and O. Straub, The low-spin black hole in LMC X-3, Astrophys. J. 793, L29 (2014).

[45] J. A. Orosz et al., A 15.65 solar mass black hole in an eclipsing binary in the nearby spiral galaxy Messier 33, Nature (London) 449, 872 (2007).

[46] J. Liu, J. E. McClintock, R. Narayan, S. W. Davis, and J. A. Orosz, Precise measurement of the spin parameter of the stellar-mass black hole M33 X-7, Astrophys. J. Lett. 679, L37 (2008).

[47] B. M. Peterson et al., Central masses and broad-line region sizes of active galactic nuclei. II. A Homogeneous analysis of a large reverberation-mapping database, Astrophys. J. 613, 682 (2004).

[48] D. J. Walton, E. Nardini, A. C. Fabian, L. C. Gallo, and R. C. Reis, Suzaku observations of 'bare' active galactic nuclei, Mon. Not. R. Astron. Soc. 428, 2901 (2013).

[49] A. M. Lohfink, C. S. Reynolds, J. M. Miller, L. W. Brenneman, R. F. Mushotzky, M. A. Nowak, and A. C. Fabian, The black hole spin and soft x-ray excess of the 
luminous Seyfert galaxy Fairall 9, Astrophys. J. 758, 67 (2012).

[50] L. C. Gallo, G. Miniutti, J. M. Miller, L. W. Brenneman, A. C. Fabian, M. Guainazzi, and C. S. Reynolds, Multiepoch x-ray observations of the Seyfert 1.2 galaxy Mrk 79: Bulk motion of the illuminating X-ray source, Mon. Not. R. Astron. Soc. 411, 607 (2011).

[51] L. W. Brenneman, C. S. Reynolds, M. A. Nowak, R. C. Reis, M. Trippe, A. C. Fabian, K. Iwasawa, J. C. Lee, J. M. Miller, R. F. Mushotzky, K. Nandra, and M. Volonteri, The spin of the supermassive black hole in NGC 3783, Astrophys. J. 736, 103 (2011).

[52] I. M. McHardy, K. F. Gunn, P. Uttley, and M. R. Goad, MCG-6-30-15: Long timescale X-ray variability, black hole mass and AGN high states, Mon. Not. R. Astron. Soc. 359, 1469 (2005).

[53] L. W. Brenneman and C. S. Reynolds, Constraining black hole spin via X-ray spectroscopy, Astrophys. J. 652, 1028 (2006).

[54] A. R. Patrick, J. N. Reeves, A. P. Lobban, D. Porquet, and A. G. Markowitz, Assessing black hole spin in deep Suzaku observations of Seyfert 1 AGN, Mon. Not. R. Astron. Soc. 416, 2725 (2011).

[55] A. R. Patrick, J. N. Reeves, D. Porquet, A. G. Markowitz, V. Braito, and A. P. Lobban, A Suzaku survey of FeK lines in Seyfert 1 active galactic nuclei, Mon. Not. R. Astron. Soc. 426, 2522 (2012).

[56] R. D. Peccei and H. R. Quinn, CP Conservation in the Presence of Instantons, Phys. Rev. Lett. 38, 1440 (1977).

[57] S. Weinberg, A New Light Boson?, Phys. Rev. Lett. 40, 223 (1978).

[58] F. Wilczek, Problem of Strong $P$ and $T$ Invariance in the Presence of Instantons, Phys. Rev. Lett. 40, 279 (1978).

[59] W. H. Press, B. S. Ryden, and D. N. Spergel, Single Mechanism for Generating Large-Scale Structure and Providing Dark Missing Matter, Phys. Rev. Lett. 64, 1084 (1990).

[60] W. Hu, R. Barkana, and A. Gruzinov, Cold and Fuzzy Dark matter, Phys. Rev. Lett. 85, 1158 (2000).

[61] D. J. E. Marsh and J. Silk, A model for halo formation with axion mixed dark matter, Mon. Not. R. Astron. Soc. 437, 2652 (2014).

[62] H.-Y. Schive, T. Chiueh, and T. Broadhurst, Cosmic structure as the quantum interference of a coherent dark wave, Nat. Phys. 10, 496 (2014).

[63] L. Hui, J. P. Ostriker, S. Tremaine, and E. Witten, Ultralight scalars as cosmological dark matter, Phys. Rev. D 95, 043541 (2017).

[64] B. S. Acharya, K. Bobkov, and P. Kumar, An M theory solution to the strong $C P$ problem and constraints on the axiverse, J. High Energy Phys. 11 (2010) 105.

[65] M. J. Stott, D. J. E. Marsh, C. Pongkitivanichkul, L. C. Price, and B.S. Acharya, Spectrum of the axion dark sector, Phys. Rev. D 96, 083510 (2017).

[66] E. Witten, Some properties of $\mathrm{O}(32)$ superstrings, Phys. Lett. B 149, 351 (1984).

[67] P. Svrcek and E. Witten, Axions in string theory, J. High Energy Phys. 06 (2006) 051.

[68] J. P. Conlon, The QCD axion and moduli stabilisation, J. High Energy Phys. 05 (2006) 078.
[69] M. Cicoli, M. D. Goodsell, and A. Ringwald, The type IIB string axiverse and its low-energy phenomenology, J. High Energy Phys. 10 (2012) 146.

[70] M. Kreuzer and H. Skarke, Complete classification of reflexive polyhedra in four-dimensions, Adv. Theor. Math. Phys. 4, 1209 (2000).

[71] R. Altman, J. Gray, Y.-H. He, V. Jejjala, and B. D. Nelson, A Calabi-Yau database: Threefolds constructed from the Kreuzer-Skarke list, J. High Energy Phys. 02 (2015) 158.

[72] A. Corti, M. Haskins, J. Nordström, and T. Pacini, $\mathrm{G}_{2}$-manifolds and associative submanifolds via semi-Fano 3-folds, Duke Math. J. 164, 1971 (2015).

[73] J. Halverson and D. R. Morrison, The landscape of M-theory compactifications on seven-manifolds with $\mathrm{G}_{2}$ holonomy, J. High Energy Phys. 04 (2015) 047.

[74] J. Halverson and D. R. Morrison, On gauge enhancement and singular limits in $\mathrm{G}_{2}$ compactifications of M-theory, J. High Energy Phys. 04 (2016) 100.

[75] A. P. Braun, Tops as building blocks for $G_{2}$ manifolds, J. High Energy Phys. 10 (2017) 083.

[76] A. P. Braun and S. Schfer-Nameki, Compact, singular G2-holonomy manifolds and M/heterotic/F-theory duality, J. High Energy Phys. 04 (2018) 126.

[77] A. P. Braun and M. Del Zotto, Mirror symmetry for $G_{2}$-manifolds: Twisted connected sums and dual tops, J. High Energy Phys. 05 (2017) 080.

[78] A. P. Braun and M. Del Zotto, Towards generalized mirror symmetry for twisted connected sum $G_{2}$ manifolds, J. High Energy Phys. 03 (2018) 082.

[79] M. R. Douglas and S. Kachru, Flux compactification, Rev. Mod. Phys. 79, 733 (2007).

[80] M. L. Mehta, Random Matrices (Academic, New York, 1991).

[81] R. Easther and L. McAllister, Random matrices and the spectrum of N-flation, J. Cosmol. Astropart. Phys. 05 (2006) 018.

[82] T. C. Bachlechner, D. Marsh, L. McAllister, and T. Wrase, Supersymmetric vacua in random supergravity, J. High Energy Phys. 01 (2013) 136.

[83] C. Long, L. McAllister, and P. McGuirk, Heavy tails in Calabi-Yau moduli spaces, J. High Energy Phys. 10 (2014) 187.

[84] C. Brodie and M. C. D. Marsh, The spectra of type IIB flux compactifications at large complex structure, J. High Energy Phys. 01 (2016) 037.

[85] T. C. Bachlechner, K. Eckerle, O. Janssen, and M. Kleban, Systematics of aligned axions, J. High Energy Phys. 11 (2017) 036.

[86] R. Brito, V. Cardoso, and P. Pani, Black holes as particle detectors: Evolution of superradiant instabilities, Classical Quantum Gravity 32, 134001 (2015).

[87] J. Aasi et al. (LIGO Scientific Collaboration), Advanced LIGO, Classical Quantum Gravity 32, 115012 (2015).

[88] P. Amaro-Seoane et al., Low-frequency gravitational-wave science with eLISA/NGO, Classical Quantum Gravity 29, 124016 (2012).

[89] P. Amaro-Seoane et al., eLISA/NGO: Astrophysics and cosmology in the gravitational-wave millihertz regime, GW Notes 6, 4 (2013). 
[90] S. Dimopoulos, P. W. Graham, J. M. Hogan, M. A. Kasevich, and S. Rajendran, Atomic gravitational wave interferometric sensor (AGIS), Phys. Rev. D 78, 122002 (2008).

[91] J. Luo et al. (TianQin Collaboration), TianQin: A spaceborne gravitational wave detector, Classical Quantum Gravity 33, 035010 (2016).

[92] B. Sathyaprakash et al., Scientific objectives of Einstein Telescope, Classical Quantum Gravity 29, 124013 (2012); Erratum, Classical Quantum Gravity30, 079501(E) (2013).

[93] S. Kawamura et al., The Japanese space gravitational wave antenna - DECIGO, J. Phys. Conf. Ser. 120, 032004 (2008).

[94] S. Detweiler, Klein-Gordon equation and rotating black holes, Phys. Rev. D 22, 2323 (1980).

[95] T. J. M Zouros and D. M. Eardley, Instabilities of massive scalar perturbations of a rotating black hole, Ann. Phys. (N.Y.) 118, 139 (1979).

[96] S. R. Dolan, Instability of the massive Klein-Gordon field on the Kerr spacetime, Phys. Rev. D 76, 084001 (2007).

[97] S. R. Dolan, Superradiant instabilities of rotating black holes in the time domain, Phys. Rev. D 87, 124026 (2013).

[98] H. Furuhashi and Y. Nambu, Instability of massive scalar fields in Kerr-Newman space-time, Prog. Theor. Phys. 112, 983 (2004).

[99] V. Cardoso and S. Yoshida, Superradiant instabilities of rotating black branes and strings, J. High Energy Phys. 07 (2005) 009.

[100] H. Yoshino and H. Kodama, The bosenova and axiverse, Classical Quantum Gravity 32, 214001 (2015).

[101] F. Shankar, D. H. Weinberg, and J. Miralda-Escude, Selfconsistent models of the AGN and black hole populations: Duty cycles, accretion rates, and the mean radiative efficiency, Astrophys. J. 690, 20 (2009).

[102] V. Cardoso, Ó. J. C. Dias, G. S. Hartnett, M. Middleton, P. Pani, and J.E. Santos, Constraining the mass of dark photons and axion-like particles through black-hole superradiance, J. Cosmol. Astropart. Phys. 03 (2018) 043.

[103] B. P. Abbott et al. (Virgo and LIGO Scientific Collaborations), GW170608: Observation of a 19 solar-mass binary black hole coalescence, Astrophys. J. Lett. 851, L35 (2017).

[104] B. P. Abbott et al. (Virgo and LIGO Scientific Collaborations), GW170814: A Three-Detector Observation of Gravitational Waves from a Binary Black Hole Coalescence, Phys. Rev. Lett. 119, 141101 (2017).

[105] A. Klein et al., Science with the space-based interferometer eLISA: Supermassive black hole binaries, Phys. Rev. D 93, 024003 (2016).

[106] J. E. McClintock, R. Narayan, and J. F. Steiner, Black hole spin via continuum fitting and the role of spin in powering transient jets, Space Sci. Rev. 183, 295 (2014).

[107] I. M. Johnstone, On the distribution of the largest eigenvalue in principal components analysis, Ann. Statist. 29, 295 (2001).
[108] S. Kachru, R. Kallosh, A. Linde, and S. P. Trivedi, de Sitter vacua in string theory, Phys. Rev. D 68, 046005 (2003).

[109] J. D. Bowman, A. E. E. Rogers, R. A. Monsalve, T. J. Mozdzen, and N. Mahesh, An absorption profile centred at 78 megahertz in the sky-averaged spectrum, Nature (London) 555, 67 (2018).

[110] A. Lidz and L. Hui, The implications of a pre-reionization $21 \mathrm{~cm}$ absorption signal for fuzzy dark matter, Phys. Rev. D 98, 023011 (2018).

[111] A. Schneider, Constraining non-cold dark matter models with the global 21-cm signal, arXiv:1805.00021 [Phys. Rev. D (to be published)].

[112] T. Helfer, D. J. E. Marsh, K. Clough, M. Fairbairn, E. A. Lim, and R. Becerril, Black hole formation from axion stars, J. Cosmol. Astropart. Phys. 03 (2017) 055.

[113] D. G. Levkov, A. G. Panin, and I. I. Tkachev, Relativistic Axions from Collapsing Bose Stars, Phys. Rev. Lett. 118, 011301 (2017).

[114] V. Desjacques, A. Kehagias, and A. Riotto, Impact of ultralight axion self-interactions on the large scale structure of the Universe, Phys. Rev. D 97, 023529 (2018).

[115] D. J. E. Marsh and A.-R. Pop, Axion dark matter, solitons and the cusp-core problem, Mon. Not. R. Astron. Soc. 451, 2479 (2015).

[116] Y.-Z. Ma, G. Hinshaw, and D. Scott, WMAP observations of Planck ESZ clusters, Astrophys. J. 771, 137 (2013).

[117] C. Long, L. McAllister, and J. Stout, Systematics of axion inflation in Calabi-Yau hypersurfaces, J. High Energy Phys. 02 (2017) 014.

[118] T. C. Bachlechner, C. Long, and L. McAllister, Planckian axions and the weak gravity conjecture, J. High Energy Phys. 01 (2016) 091.

[119] T. C. Bachlechner, C. Long, and L. McAllister, Planckian axions in string theory, J. High Energy Phys. 12 (2015) 042.

[120] J. Halverson and P. Langacker, TASI Lectures on Remnants from the String Landscape, in Theoretical Advanced Study Institute in Elementary Particle Physics: Physics at the Fundamental Frontier (TASI 2017), Boulder, CO, USA, 2017 (to be published), arXiv:1801.03503.

[121] T. C. Bachlechner, M. Dias, J. Frazer, and L. McAllister, Chaotic inflation with kinetic alignment of axion fields, Phys. Rev. D 91, 023520 (2015).

[122] J. Baik, G. B. Arous, and S. Peche, Phase transition of the largest eigenvalue for non-null complex sample covariance matrices, arXiv:math/0403022.

[123] A. Bloemendal and B. Virág, Limits of spiked random matrices I, Probab. Theory Relat. Fields 156, 795 (2013).

[124] M. Y. Mo, The rank 1 real Wishart spiked model, arXiv: 1101.5144 .

[125] B. Acharya, K. Bobkov, G. Kane, P. Kumar, and D. Vaman, An M Theory Solution to the Hierarchy Problem, Phys. Rev. Lett. 97, 191601 (2006).

[126] B. S. Acharya, K. Bobkov, G. L. Kane, P. Kumar, and J. Shao, Explaining the electroweak scale and stabilizing moduli in M theory, Phys. Rev. D 76, 126010 (2007). 